\title{
Proca Metamaterials, Massive Electromagnetism, and Nonlocality
}

This paper was downloaded from TechRxiv (https://www.techrxiv.org).

\section{LICENSE}

CC BY 4.0

SUBMISSION DATE / POSTED DATE

$13-01-2021 / 16-01-2021$

CITATION

Mikki, Said (2021): Proca Metamaterials, Massive Electromagnetism, and Nonlocality. TechRxiv. Preprint. https://doi.org/10.36227/techrxiv.13567529.v1

$\mathrm{DOI}$

10.36227/techrxiv.13567529.v1 


\title{
Proca Metamaterials, Massive Electromagnetism, and Nonlocality
}

\author{
Said Mikki
}

\begin{abstract}
We investigate a new type of electromagnetic metamaterials (MTMs), which we dub Proca MTMs, constituting an interesting medium behaving like a "relativistic material" for potential use in electromagnetic applications. It is rigorously proved using a field-theoretic approach that Maxwell theory inside certain classes of nonlocal metamaterials is equivalent to Maxwell-Proca theory in vacuum, where in the latter photons acquire a nonzero mass (massive electromagnetism.) It turns out that the key to the operation of Proca MTM is nonlocality (here spatial dispersion since the Proca MTM is homogeneous), and hence Proca MTMs represent an important example of the more general family of nonlocal MTMs. Our analysis involves multiphysics aspects, utilizing concepts and methods taken from classical electromagnetism, special relativity, quantum theory, electromagnetic materials, and antenna theory. Extensive discussion of the physics, computational methods, and design parameters of Proca MTMs is provided to further understand the nature of massive electromagnetism in nonlocal MTMs. Proca waves carry an additional polarization degree of freedom and each wave appears to behave like a single mode with two transverse components and one longitudinal. This opens the door for applications in wireless communications and other fields where information could be encoded in polarization. As a concrete application, we develop the main ingredients of Proca antennas as an example of the emerging technology of nonlocal antennas, where we establish that a single Proca dipole possesses a perfect isotropic radiation pattern, a radical departure from conventional local antennas (radiators in vacuum and temporally dispersive media) where such radiation characteristics is impossible. Moreover, the new connection between electromagnetic theory in some nonlocal MTMs and Maxwell-Proca theory allows the use of relativistic techniques developed in the latter in order to efficiently perform calculations like field quantization in nonlocal domains which would be very difficult to perform otherwise. ${ }^{1}$
\end{abstract}

Index Terms-Metamaterials, nonlocality, massive electromagnetism, antennas.

\section{INTRODUCTION}

In Proca-Maxwell theory (massive electromagnetism), the quantization of the electromagnetic field leads to photons with nonzero mass with dispersion relations fully reflecting the relativistic energy-momentum law. Since its formulation in the early 1930s by A. Proca, the theory has attracted attention in both fundamental theory and applications. However, in recent years the subject has exploded in various fields of physics and engineering. Most of the investigations focus on the mechanism of acquiring mass in photon fields and the corresponding dispersion relations. In this paper we report a radically new approach where an exact analogy is established between

\footnotetext{
${ }^{1}$ The author is with the Zhejiang University/University of Illinois at UrbanaCampaign (ZJU-UIUC) Institute, Haining, Zhejiang, China, and the ECE department of Royal Military College of Canada, Kingston, Canada. He can be reached at said.m.mikki@gmail.com.
}

massive electromagnetism and certain classes of metamaterials (a special nonlocal medium we call Proca Metamaterial). Our key findings illustrate the essential role played by the electromagnetic (EM) nonlocality of the special Proca homogeneous and isotropic material domain's response (spatial dispersion) in securing the exact field-theoretic correspondence between Proca fields in vacuum on one hand, and Maxwellian fields in Proca metamaterial (MTM) on another. In this way, the well-known theory of vacuum Proca fields can be deployed to solve computationally difficult problems in nonlocal MTMs. Conversely, the Proca MTM discovered here can be used as a practical model to understand the fundamental physics of Proca fields both computationally and experimentally since such nonlocal MTM might be constructed in the lab.

Proca MTMs exhibit new electromagnetic behaviour, such as nonzero photon mass, the existence of additional longitudinal waves, energy/momentum localization, and many others. Before engaging in a further elaboration of our main findings and their potential significance for applications, we first provide a literature view on the subject.

For elementary approaches to Proca theory and photon mass concepts in general, see [1]-[4]. The subject of Proca equation and massive electromagnetism is also discussed in textbooks on field theory such as [5]-[8]. ${ }^{2}$ When the photon mass $m_{\mathrm{ph}}$ vanishes, we work with conventional "massless" electromagnetic theory, i.e., Maxwell theory. On the other hand, when $m_{\mathrm{ph}} \neq 0$, qualitatively new phenomena occur, most famously the existence of longitudinal polarization in photons propagating in vacuum [8]. Since there is no conclusive experimental evidence that such polarization has been observed in vacuum [10], [11], Proca theory was not considered applicable to vacuum fields. However, search for experimental evidence of nonzero photon mass has never lost momentum and in general there is no universally-accepted proof that the photon mass in vacuum must be zero [10]. Moreover, Proca theory did motivate the creation of the massive boson theory in quantum field theory, which lies behind the electroweak force unification of nuclear weak and electromagnetic interactions and is now a standard topic in quantum field theory (QFT) [7]. In addition, Proca theory has been widely applied to general relativity, cosmology, and quantum gravity (see references quoted below).

Even though Proca theory is nearly a ninety-year old research area, in recent years the subject has experienced an explosive growth in terms of publications dedicated to

\footnotetext{
${ }^{2}$ Note that the more well-known concept of "electromagnetic mass" [5] can be discussed independently of photon mass and some authors presented arguments supporting the claim that EM mass can be understood even with zero photon mass, e.g., see [9].
} 
various possible applications. This includes fundamental reexaminations of its theoretical foundations such as [12] [14]. Applications of Proca theory include most prominently general relativity and cosmology, where in the last ten years it has experienced very extensive growth, e.g., see [15] [20]. Propagation of electromagnetic waves in waveguides implies nonzero photon mass even if the waveguide is not filled with any material [2], [21]. Here, the source of the effective mass is the boundary conditions imposed on the fields inside the guiding structure. Ultimately, this is due to the existence of standing modes since it has been shown that the presence of such waves requires nonzero photon mass [22]. Moreover, light propagation in periodic structures, e.g., photonic crystals, was found to lead to the emergence of a nonzero effective mass. This is similar to the effectivemass approximation in condensed-matter physics [23], which is often based on band structure analysis [24]. Heavy photons have been reported theoretically and experimentally in some photonic crystals [25]. Similarities between light propagation and the dynamical motion of particles with negative mass was observed in the analysis of hyperbolic metamaterials [26], [27]. Moreover, metamaterials in general and hyperbolic metamaterials in particular were used as models to understand cosmological structures [28]. Tachyonic particles, i.e., particles with imaginary mass [29], [30], are equivalent to certain electromagnetic modes in hyperbolic materials and photonic crystals (massive photons with imaginary mass) [28] and have also been investigated in homogeneous dispersive media [31].

There is then no essential reason why one should restrict the analysis of massive electromagnetism to the case of the vacuum since photons may in fact acquire mass through interactions with complex material domains. This has already been reported in numerous researches on electromagnetic wave propagation in media such as plasma [32]-[35], nonlinear crystals [36], time-derivative Lorentzian media [37], fluids [38], photonic crystals [39], [40], quantum gases [41].

In this paper we approach the subject of massive electromagnetism from a different perspective compared with many other works. We list below some of the distinctive features in our take:

1) We work within exact field-theoretic framework, deriving using rigorous analysis the field-based equivalence of Maxwell-Proca theory and vacuum and Maxwell theory in a special MTM (the Proca MTM.) Maxwell theory in Proca MTMs turns out to be exactly equivalent to Maxwell-Proca theory in vacuum. This equivalence goes beyond the final dispersion relations and applies to all components of the full vectorial EM fields without exception.

2) We emphasize the fundamental role plated by EM nonlocality (here spatial dispersion) in securing the equivalence above between vacuum Proca and MTM theories.

3) The nonzero mass of photons associated with waves propagating in Proca MTMs is an important design parameter that can often be measured. This will be exploited to provide various examples and illustrations.

4) We introduce the concept of Proca waves as EM waves

\begin{tabular}{cl}
\hline \hline & Definitions of Various Concepts \\
\hline \hline Proca Wave & The classical electromagnetic wave underlying the \\
& quantized (massive) photons described by Proca's \\
theory characterized by longitudinal wave compo- & nent that is inherently coupled with the other two \\
& transverse polarization components. The three polar- \\
& ization components together constitute a combined \\
& object living in the 4-dimensional Minkowsky space \\
& of SR and are generated by the Lorentz group. \\
Massive Photon & A quantized state of electromagnetic radiation in \\
& which photons acquire mass satisfying the massive \\
& relativistic particle dispersion relation. Massive pho- \\
tons are the quantum version of Proca waves. & An isotropic and homogeneous nonlocal material do- \\
main with specialized temporal and spatial dispersion & profiles such that a Proca wave can be excited in \\
this domain or a massive photon may exist in the \\
medium. \\
A nonlocal radiating system comprised of an exter- \\
nally applied source embedded into a Proca metama- \\
terial capable of radiating a single Proca wave mode \\
with three degrees of polarization (two transverse \\
and one longitudinal) generated by a subgroup of \\
the Lorentz group.
\end{tabular}

TABLE I

Main Concepts Discussed In THIS PAPER

possessing three degrees of polarization and probe in depth their properties.

5) We propose new applications in the emerging field of nonlocal antenna theory. Proca antennas can be perfectly isotopic, in direct contrast to conventional antennas.

The author believes that the multidisciplinary and multifaceted subject of massive EM in Proca MTMs is expected to help stimulate a host of new applications in both theory and experiments, especially in the areas of multiphysics and electromagnetic systems. For example, it has the potential to help establishing alternative circuit and antenna technologies harnessing the new behaviour of EM waves possessing massive photons.

Finally, while our focus in the present work will be on the essential physics and the computational methods and applications connected with them, we also mention some of the possible connections with basic research. Indeed, for fundamental applications, we note the history of attempts to phenomenologically model the quantum vacuum as an optical medium via the Heisenberg-Euler effective action approach [42]-[44]. In such models, one can recover the observable physics in vacuum but now derived using methods that are essentially that of conventional optics [45]. In this connection, the discovery of a special nonlocal material (Proca MTM) that is equivalent to Maxwell-Proca theory may open the door for new insight into the fundamental physics of elementary particles and photons in vacuum.

The main concepts introduced and discussed in this paper are summarized in Table I. Some of the needed background relations or new ones derived in this paper are accumulated in Table II. A list of frequently used acronyms is placed in Table III. 


\begin{tabular}{ll}
\hline \multicolumn{1}{c}{ Quantity } & \multicolumn{1}{c}{ Expression } \\
\hline$E_{\mathbf{p}}$ (photon energy) & $E_{\mathbf{p}}=\hbar \omega$ \\
$\mathbf{p}$ (photon momentum) & $\mathbf{p}=\hbar \mathbf{k}$ \\
$m$ (normalized photon mass) & $m=m_{\mathrm{ph}} c / \hbar$ \\
$E_{\mathrm{ph}}$ (photon mass energy) & $E_{\mathrm{ph}}=m_{\mathrm{ph}} c^{2}$ \\
$p^{\mu}$ (photon 4-momentum vector) & $p^{\mu}=\left(E_{\mathbf{p}} / c, \mathbf{p}\right)$ \\
$\mathbf{v}_{p}$ (wave phase velocity) & $\mathbf{v}_{p}=(\omega / k) \hat{k}$ \\
$\mathbf{v}_{g}$ (wave group velocity) & $\mathbf{v}_{g}=\nabla_{\mathbf{k}} \omega$ \\
$\mathbf{v}_{\mathrm{ph}}$ (relativistic photon velocity) & $\mathbf{v}_{\mathrm{ph}}=c^{2} \mathbf{p} / E$ \\
$\omega_{\mathrm{ph}}$ (photon mass frequency) & $\omega_{\mathrm{ph}}=E_{\mathrm{ph}} / \hbar=m c$ \\
$\omega_{\mathrm{p}}$ (Proca MTM resonance frequency) & $\omega_{\mathrm{p}}=c \sqrt{m^{2}+k^{2}}$ \\
$\lambda_{\mathrm{ph}}$ (photon mass wavelength) & $\lambda_{\mathrm{ph}}=2 \pi / m$ \\
$k_{\mathrm{ph}}$ (photon mass wavenumber) & $k_{\mathrm{ph}}=2 \pi / \lambda_{\mathrm{ph}}=m$ \\
\hline
\end{tabular}

TABLE II

LIST OF BASIC RELATIONS USED IN THIS PAPER

\begin{tabular}{ll}
\hline Acronym & \multicolumn{1}{c}{ Meaning } \\
\hline EM & Electromagnetics/Electromagnetic \\
MTM & Metamaterial \\
L/T & Longitudinal/Transverse \\
SR & Special Relativity \\
\hline
\end{tabular}

TABLE III

LIST OF ABBREVIATIONS USED IN THIS PAPER

\section{The MAXWELl-Proca THeORY}

The normalized mass $m$ is the key parameter in Proca theory, which is given in terms of the photon mass $m_{\mathrm{ph}}$ through the relation [5], [7], [10]

$$
m=\frac{m_{\mathrm{ph}} c}{\hbar},
$$

where $\hbar$ is the reduced Plank constant and $c$ the speed of light. The parameter $m$ has the units of inverse length (in SI we use $\mathrm{m}^{-1}$ ), and hence the quantity $1 / m$ is a characteristic length scale. For later convenience, we define the photon mass characteristic wavelength, which we denote by $\lambda_{\mathrm{ph}}$, through the relation

$$
\lambda_{\mathrm{ph}}:=\frac{2 \pi}{m},
$$

which allows us to express the normalized photon mass $m$ by $m=2 \pi / \lambda_{\mathrm{ph}}$, an expression identical to the wavenumber $k=2 \pi / \lambda$ since both $m$ and $k$ share the same units and tend to be summed together in the same expressions. The photon mass (and consequently $m$ and $\lambda_{\mathrm{ph}}$ ) are in general complex, with emphasis usually put on the two cases of real effective photon mass and imaginary mass, i.e., $m^{2}>0$ and $m^{2}<$ 0 , respectively. The imaginary photon mass for example can be observed in numerical simulation, fundamental derivations [28], and experiments [31].

The essential motivation behind Proca's modification of Maxwell's field equation was to derive a relativistic field equations that parallel the Klein-Gordon's equation for particles [8]. To accomplish this, Proca added two terms to two of Maxwell's equations, while keeping the rest the same. The dynamic field variable is the 4-vector potential $A^{\mu}:=(\varphi, \mathbf{A})$, where $\varphi$ and $\mathbf{A}$ are the electric (scalar) potential and the magnetic (vector) potentials, respectively. In the vector form, Maxwell-Proca equations are [10]:

$$
\begin{gathered}
\nabla \times \mathbf{B}(\mathbf{r}, t)=\mu_{0} \varepsilon_{0} \frac{\partial \mathbf{E}(\mathbf{r}, t)}{\partial t}+\mu_{0} \mathbf{J}(\mathbf{r}, t)-m^{2} \mathbf{A}(\mathbf{r}, t), \\
\nabla \cdot \mathbf{E}(\mathbf{r}, t)=\frac{1}{\varepsilon_{0}} \rho(\mathbf{r}, t)-m^{2} \varphi(\mathbf{r}, t), \\
\nabla \times \mathbf{E}(\mathbf{r}, t)=-\frac{\partial \mathbf{B}(\mathbf{r}, t)}{\partial t}, \quad \nabla \cdot \mathbf{B}(\mathbf{r}, t)=0, \\
\mathbf{E}(\mathbf{r}, t)=-\nabla \phi(\mathbf{r}, t)-\frac{\partial \mathbf{A}(\mathbf{r}, t)}{\partial t}, \mathbf{B}(\mathbf{r}, t)=\nabla \times \mathbf{A}(\mathbf{r}, t) .
\end{gathered}
$$

Using the standard procedure of radiation potentials [5], it is straight forward to show from the modified Maxwell-Proca equations that the scalar and vector potential problems are decoupled with fundamental field equations given by [8], [10]

$$
\begin{gathered}
\left(\nabla^{2}-\frac{1}{c^{2}} \frac{\partial^{2}}{\partial t^{2}}\right) \varphi(\mathbf{r}, t)-m^{2} \varphi(\mathbf{r}, t)=-\frac{\rho(\mathbf{r}, t)}{\varepsilon_{0}}, \\
\left(\nabla^{2}-\frac{1}{c^{2}} \frac{\partial^{2}}{\partial t^{2}}\right) \mathbf{A}(\mathbf{r}, t)-m^{2} \mathbf{A}(\mathbf{r}, t)=-\mu_{0} \mathbf{J}(\mathbf{r}, t) .
\end{gathered}
$$

The Proca field equations (7) and (8) are very similar to the standard Maxwell's field equation with exception of the linear terms proportional to $m^{2} \varphi$ and $m^{2} \mathbf{A}$, respectively.

In order to understand the logic behind Proca theory, we note that the four scalar equations comprising (7) and (8) each has the form of the Klein-Gordon equation [7], [8]

$$
\left(\nabla^{2}-\frac{1}{c^{2}} \frac{\partial^{2}}{\partial t^{2}}\right) \psi(\mathbf{r}, t)-m^{2} \psi(\mathbf{r}, t)=0
$$

i.e., the simplest second-order relativistic equation of material particles. For a single wave mode of the form $\psi(\mathbf{r}, t)=$ $\exp [i(\mathbf{k} \cdot \mathbf{r}-\omega t)]$, the Klein-Gordon-type equations (7) and (8) and (9) yield

$$
E_{\mathbf{p}}^{2}=|\mathbf{p}|^{2} c^{2}+m_{\mathrm{ph}}^{2} c^{4},
$$

where $\mathbf{p}=\hbar \mathbf{k}$ is the 3 -momentum, $E_{\mathbf{p}}=\hbar \omega$ is the photon energy at the momentum state $\mathbf{p}$, and the normalized mass expression (1) was used. Since the 4-momentum vector $p^{\nu}, \nu=1,2,3,4$, is $p:=(E / c, \mathbf{k})$, where $E$ is the particle energy, the equation (10) is clearly the relativistic dispersion relation of a particle with mass $m_{\mathrm{ph}}$, suggesting that the Maxwell-Proca equations model a relativistic photon field particle with momentum $p$, mass $m_{\mathrm{ph}}$, and energy $E_{\mathrm{ph}}$ related by the familiar relativistic formula. ${ }^{3}$ It is precisely for obtaining this dispersion law that Proca had initially invented his original theory.

It is important at this point to point out another major difference between Maxwell's and Proca's electromagnetic theories. It can be shown that the Lorenz gauge

$$
\nabla \cdot \mathbf{A}(\mathbf{r}, t)+\frac{1}{c^{2}} \frac{\partial \varphi(\mathbf{r}, t)}{\partial t}=0
$$

needed to write the Maxwell-Proca field equations (3)-(8) is the only possible gauge. In other words, the modification of Maxwell's equations entitled by adding two mass terms to

\footnotetext{
${ }^{3}$ That is, $p_{\nu} p^{\nu}=m^{2} c^{2}$ using the repeated index notation for implied sums where $m$ is the particle's mass [5], [46].
} 
the equations (3) and (4) necessarily implies for reason due to internal consistency that no gauge other than (11) is used. Therefore, Proca field theory is not gauge invariant, and the local gauge symmetry group is not applicable to this type of theories [7], [8].

\section{An Exact Model for Maxwell-Proca Massive ELECTROMAgnetism THROUgh NONLOCAL METAMATERIALS}

In this section, we demonstrate the ability to design a special type of nonlocal domains inside which the field equations become formally identical to (3)-(8). This model is exact and no approximations are involved. Moreover, the equivalence between the the vacuum Maxwell-Proca theory and MaxwellProca-MTM theory is based on the fields, not merely the final dispersion relations.

\section{A. Preliminary Considerations: The Theory of Nonlocal Meta- materials}

Let us first define the terminologies involved in our problem field. In this paper, by the term nonlocal material we mean a spatially-dispersive homogeneous and time-invariant domain [47]-[49]. Such materials can be described by a material tensor response, e.g., conductivity or dielectric tensors, which generally come out in the form $\overline{\mathbf{R}}(\mathbf{k}, \omega)$ [50]-[52]. The dependence on the temporal frequency $\omega$ is often termed temporal dispersion or just dispersion in literature and is the most widely discussed type of material behaviour. On the other hand, dependence on $\mathbf{k}$ leads to the so-called spatial dispersion effect [47]. Nonlocal metamaterials are defined as nonlocal materials engineered by the designer to attain certain electromagnetic performance measures [49]. By design of a nonlocal MTM we mean finding the response function (conductivity, susceptibility, dielectric function, etc) such that the desired electromagnetic response is attained with applications in MTMs, nanotechnology, and nano-electromagnetism [53][64]. Finally, by realization of a nonlocal MTM we mean building an actual material configuration, normally assembled from its microscopic constituents, such that its effective response function will approximate the response functions obtained through the previously described process of material design [49], [51], [53], [65].

The most convenient formalism for working with nonlocal MTMs within the framework of linear response theory (our main scope here) is the frequency-momentum space representation (momentum space for short) [48], [54], [55], [66], [67]. Here, the spacetime fields and currents are transformed into the 4-dimensional Fourier space with Minkoweskian structure given as follows:

$$
\begin{gathered}
\mathbf{F}(\mathbf{k}, \omega):=\int_{\mathbb{R}^{4}} \mathrm{~d}^{3} r \mathrm{~d} t \mathbf{F}(\mathbf{r}, t) e^{-\mathrm{i} \mathbf{k} \cdot \mathbf{r}+\mathrm{i} \omega t}, \\
\mathbf{F}(\mathbf{r}, t)=\int_{\mathbb{R}^{4}} \frac{\mathrm{d}^{3} k \mathrm{~d} \omega}{(2 \pi)^{4}} \mathbf{F}(\mathbf{k}, \omega) e^{\mathrm{i} \mathbf{k} \cdot \mathbf{r}-\mathrm{i} \omega t} .
\end{gathered}
$$

Maxwell's equations in a material domain can be written as

$$
\begin{gathered}
\nabla \times \mathbf{E}(\mathbf{r}, t)=-\frac{\partial \mathbf{B}(\mathbf{r}, t)}{\partial t}, \\
\nabla \times \mathbf{B}(\mathbf{r}, t)=\frac{1}{c^{2}} \frac{\partial \mathbf{E}(\mathbf{r}, t)}{\partial t}+\mu_{0} \mathbf{J}(\mathbf{r}, t), \\
\nabla \cdot \mathbf{E}(\mathbf{r}, t)=\frac{1}{\varepsilon_{0}} \rho(\mathbf{k}, \omega), \quad \nabla \cdot \mathbf{B}(\mathbf{r}, t)=0 .
\end{gathered}
$$

The transformed Maxwell's equations in momentum space then become

$$
\begin{gathered}
\mathbf{k} \times \mathbf{E}(\mathbf{k}, \omega)=\omega \mathbf{B}(\mathbf{k}, \omega), \\
\mathrm{i} \mathbf{k} \times \mathbf{B}(\mathbf{k}, \omega)=\mu_{0} \mathbf{J}(\mathbf{k}, \omega)-\frac{\mathrm{i} \omega}{c^{2}} \mathbf{E}(\mathbf{k}, \omega), \\
\mathbf{k} \cdot \mathbf{E}(\mathbf{k}, \omega)=\frac{-\mathrm{i}}{\varepsilon_{0}} \rho(\mathbf{k}, \omega), \quad \mathbf{k} \cdot \mathbf{B}(\mathbf{k}, \omega)=0 .
\end{gathered}
$$

In the linear theory of electromagnetic materials [67], we may decompose the current and charge densities into external and induced parts

$$
\begin{aligned}
& \mathbf{J}(\mathbf{k}, \omega)=\mathbf{J}_{\mathrm{ex}}(\mathbf{k}, \omega)+\mathbf{J}_{\text {ind }}(\mathbf{k}, \omega), \\
& \rho(\mathbf{k}, \omega)=\rho_{\mathrm{ex}}(\mathbf{k}, \omega)+\rho_{\text {ind }}(\mathbf{k}, \omega),
\end{aligned}
$$

respectively. In what follows, we assume source-free materials, i.e., $\rho_{\mathrm{ex}}(\mathbf{k}, \omega)=0, \mathbf{J}_{\mathrm{ex}}(\mathbf{k}, \omega)=0$. The current response is given by

$$
\mathbf{J}_{\text {ind }}(\mathbf{k}, \omega)=\overline{\bar{\sigma}}(\mathbf{k}, \omega) \cdot \mathbf{E}(\mathbf{k}, \omega)
$$

where $\overline{\bar{\sigma}}(\mathbf{k}, \omega)$ is the nonlocal conductivity profile of the medium. The field response is usually written as

$$
\mathbf{D}(\mathbf{k}, \omega)=\overline{\bar{\varepsilon}}(\mathbf{k}, \omega) \cdot \mathbf{E}(\mathbf{k}, \omega),
$$

where

$$
\overline{\bar{\varepsilon}}(\mathbf{k}, \omega)=\overline{\mathbf{I}}+\frac{\mathrm{i}}{\omega \varepsilon_{0}} \overline{\bar{\sigma}}(\mathbf{k}, \omega)
$$

is the generalized equivalent (normalized) dielectric function. Note that in contrast to the conventional dielectric function in the multipole approach to material response, the tensor $\overline{\bar{\varepsilon}}(\mathbf{k}, \omega)$ does include magnetic effects, not only the electric ones. ${ }^{4}$

\section{B. Proca Metamaterials: Derivation of the Equivalent Nonlo- cal Tensor Response Function}

Our main task here is to derive a special material response function $\overline{\bar{\varepsilon}}(\mathbf{k}, \omega)$ such that the system of Maxwell's equation given by (14) becomes formally equivalent to the MaxwellProca system (3)-(5). To achieve this, first note that the source current and charge densities $\mathbf{J}$ and $\rho$ appearing in the latter system are entirely due to external sources, i.e., $\rho(\mathbf{r}, t)=\rho_{\mathrm{ex}}(\mathbf{r}, t), \mathbf{J}(\mathbf{r}, t)=\mathbf{J}_{\mathrm{ex}}(\mathbf{r}, t)$. Setting these to zero (the same will be done with the electrodynamics of nonlocal MTMs prescribed by (14)), the relation (4) then implies

$$
\varphi(\mathbf{r}, t)=\frac{-1}{m^{2}} \nabla \cdot \mathbf{E}(\mathbf{r}, t)
$$

\footnotetext{
${ }^{4}$ In fact, spatial dispersion can arise from the inclusion of the magnetic response alone. In this Fourier (momentum space) formalism, a multipole response described by $\varepsilon(\omega)$ and $\mu(\omega)$ is translated into a material response function $\overline{\bar{\varepsilon}}(\mathbf{k}, \omega)$ with spatial dispersion. See for example [50], [51], [68]. Even for isotropic media, the response function in momentum space is a dyadic (tensorial) quantity.
} 
where it is assumed from now on that $m \neq 0$.

Therefore, the electric potential $\varphi(\mathbf{r}, t)$ is completely determined by the divergence of the electric field, which in turns says that only the longitudinal component of the electric field contributes to $\varphi(\mathbf{r}, t)$. Let us now use this fact to express the magnetic potential $\mathbf{A}$ in terms of the electric field only. Using (22) in the first relation in (6), we arrive at

$$
\begin{gathered}
\left(1-\frac{1}{m^{2}} \nabla \nabla \cdot\right) \mathbf{E}(\mathbf{r}, t)=-\frac{\partial \mathbf{A}(\mathbf{r}, t)}{\partial t}, \\
\mathbf{A}(\mathbf{r}, t)=-\int \mathrm{d} t\left(1-\frac{1}{m^{2}} \nabla \nabla \cdot\right) \mathbf{E}(\mathbf{r}, t),
\end{gathered}
$$

which shows that in Proca field theory the electric potential can be completely eliminated from the problem. In particular, if the magnetic potential $\mathbf{A}(\mathbf{r}, t)$ alone is known, then the first differential equation in (23) can be solved to yield $\mathbf{E}(\mathbf{r}, t)$. Conversely, if $\mathbf{E}(\mathbf{r}, t)$ is known, we can compute the magnetic potential $\mathbf{A}(\mathbf{r}, t)$, which may be calculated by direct-time integration according to the second equation in (23). ${ }^{5}$

Transforming (23) into momentum space, the following purely algebraic (dyadic) equation is obtained:

$$
\mathbf{A}(\mathbf{k}, \omega)=\frac{1}{\mathrm{i} \omega}\left(\overline{\mathbf{I}}+\frac{k^{2}}{m^{2}} \hat{k} \hat{k}\right) \cdot \mathbf{E}(\mathbf{k}, \omega),
$$

where $\overline{\mathbf{I}}$ is the unit dyad and the unit vector $\hat{k}$ is defined by $\hat{k}:=\mathbf{k} / k$. Conversely, the electric field can be expressed in terms of the vector potential via

$$
\mathbf{E}(\mathbf{k}, \omega)=\mathrm{i} \omega\left(\overline{\mathbf{I}}+\frac{k^{2}}{m^{2}} \hat{k} \hat{k}\right)^{-1} \cdot \mathbf{A}(\mathbf{k}, \omega),
$$

where here $(\cdots)^{-1}$ signifies the inverse dyadic operation [69], [70], in this case equivalent to inverting $3 \times 3$ matrices.

Let us focus now on obtaining the equation (3) in Proca's theory by means of transforming the corresponding relation in the conventional massless Maxwell's theory (14). The medium's response in the $\nabla \times \mathbf{B}$ equation is manifested through the term $\mu_{0} \mathbf{J}$. Therefore, in order to formally establish an equivalence between Proca theory in vacuum and Maxwell's theory in our nonlocal MTM, we require that

$$
\mu_{\mathrm{o}} \mathbf{J}_{\text {ind }}(\mathbf{k}, \omega)=-m^{2} \mathbf{A}(\mathbf{k}, \omega)=\mu_{\mathrm{o}} \overline{\bar{\sigma}}(\mathbf{k}, \omega) \cdot \mathbf{E}(\mathbf{k}, \omega),
$$

which after using either (24) or (25) gives us

$$
\overline{\bar{\sigma}}(\mathbf{k}, \omega)=\frac{m^{2}}{-\mathrm{i} \omega \mu_{0}}\left(\overline{\mathbf{I}}+\frac{k^{2}}{m^{2}} \hat{k} \hat{k}\right) .
$$

This is the main conductivity tensor expression for Proca metamaterials valid for $m \neq 0$.

It is more instructive to work with the corresponding dielectric tensor form, which can be easily obtained by means of the formula (21), giving rise to

$$
\overline{\bar{\varepsilon}}(\mathbf{k}, \omega)=\left(1-\frac{m^{2} c^{2}}{\omega^{2}}\right) \overline{\mathbf{I}}-\frac{k^{2} c^{2}}{\omega^{2}} \hat{k} \hat{k} .
$$

\footnotetext{
${ }^{5}$ Note that equations (23) break down when $m=0$, clearly indicating that such gauge-dependent behaviour is not transferable to the conventional gauge-invariant Maxwell's theory by means of the limit $m \rightarrow 0$. A further discussion of the zero-mass limit is provided in Appendix A.
}

Therefore, we have proved that nonlocal metamaterials with a dielectric tensor in momentum space given by (28) support electromagnetic processes identical to those experienced by a vacuum supporting Maxwell-Proca equations (3)-(6). Note that the derivation of (28) requires $m \neq 0$. On the other hand, the dispersion relation (10) is valid for any value of $m$.

\section{Understanding the Physics of Proca Metamaterials}

\section{A. General Observations on the Proca Material Domain}

The general dielectric tensor expression (28) corresponds to an isotropic material since it can be further analyzed if we put it in the standard form used in the theory of isotropic nonlocal metamaterials. Indeed, using the identity $\overline{\mathbf{I}}=(\overline{\mathbf{I}}-\hat{k} \hat{k})+\hat{k} \hat{k}$, the expression (28) may be rewritten as

$$
\overline{\bar{\varepsilon}}(\mathbf{k}, \omega)=\underbrace{\varepsilon^{\mathrm{T}}(k, \omega)(\overline{\mathbf{I}}-\hat{k} \hat{k})}_{\text {Transverse data }}+\underbrace{\varepsilon^{\mathrm{L}}(k, \omega) \hat{k} \hat{k}}_{\text {Longitudinal data }},
$$

where

$$
\begin{aligned}
\varepsilon^{\mathrm{T}}(k, \omega):=1-\frac{\omega_{\mathrm{p}}^{2}(0)}{\omega^{2}} & =1-\frac{m^{2} c^{2}}{\omega^{2}}=1-\frac{m_{\mathrm{ph}}^{2} c^{4}}{h^{2} \omega^{2}}, \\
\varepsilon^{\mathrm{L}}(k, \omega):=1-\frac{\omega_{\mathrm{p}}^{2}(k)}{\omega^{2}} & =1-\frac{c^{2}}{\omega^{2}}\left(k^{2}+m^{2}\right) \\
& =1-\frac{c^{2}}{\omega^{2}}\left(k^{2}+\frac{m_{\mathrm{ph}}^{2} c^{2}}{h^{2}}\right),
\end{aligned}
$$

are the transverse $(\mathrm{T})$ and longitudinal $(\mathrm{L})$ dielectric functions, respectively. The quantity

$$
\omega_{\mathrm{p}}(k):=c \sqrt{k^{2}+m^{2}}=m c \sqrt{1+\left(\frac{\lambda_{\mathrm{ph}}}{\lambda}\right)^{2}}
$$

plays the role of a wavenumber-dependent "plasma frequency" characterizing the Drude-like temporal dispersion profile (more on this shortly) exhibited by the Proca MTM dielectric function (28). In writing the second equality in (32), the definition of the characteristic photon mass length scale given by (2) was used.

Physically, the tensor component of (29)

$$
\overline{\bar{\varepsilon}}^{\mathrm{T}}(\mathbf{k}, \omega):=\varepsilon^{\mathrm{T}}(\mathbf{k}, \omega)(\overline{\mathbf{I}}-\hat{k} \hat{k})
$$

gives the amount of the Proca MTM total response function that is solely due to the transverse components of the applied field. Similarly, the part

$$
\overline{\bar{\varepsilon}}^{\mathrm{L}}(\mathbf{k}, \omega):=\varepsilon^{\mathrm{L}}(\mathbf{k}, \omega) \hat{k} \hat{k}
$$

quantifies only how the medium is responding to the longitudinal components of the applied electric field. This decomposition into $\mathrm{T}$ and $\mathrm{L}$ modes is made possible by the fact that every electric field $\mathbf{E}$ can be decomposed into longitudinal part $\mathbf{E}^{\mathrm{L}}$ and transverse part $\mathbf{E}^{\mathrm{T}}$ in both spacetime and moment space [50], [52], [67].

We first note the following distinctive features of the Proca MTM that might be quickly discerned after a glance at its dielectric tensor expression (29):

1) Isotropic response for both the $T$ and $L$ parts. 
2) The $T$ response exhibits only temporal dispersion.

3) The L response exhibits both temporal and spatial dispersion. Hence, only the L response is nonlocal.

4) For a fixed $k$, all temporal dispersion profiles are Drudelike for all frequencies $\omega$.

5) The nonlocal response has only one term quadratic in $k$, i.e., the factor $k^{2}+m^{2}$.

6) Spatial dispersion is not "naturally weak" in the sense that there is no fixed natural parameter measuring the strength of spatial dispersion as in natural crystals and plasma. Instead, rewriting $k^{2}+m^{2}$ as $m^{2}\left[1+\left(1 / m^{2}\right)\right] k^{2}$, the parameter $1 / m^{2}$ may be considered as the strength of spatial dispersion in this metamaterial, which is valid since in our derivation of (28) we made the mandatory assumption $m \neq 0$. Proca MTMs are then examples of nonlocal media with strong spatial dispersion whose nonlocality spatial scale can be engineered by changing $m$.

7) Both the $T$ and $L$ responses are lossless for pure real or pure imaginary photon mass parameter $m$.

We will next unpack some of the above mentioned general observations with a series of quantitative examples and include further analysis.

\section{B. Basic Representative Examples}

Fig. 1 gives the $\mathrm{T}$ and $\mathrm{L}$ response function of the Proca MTM as function of applied field frequency $\omega$ when the photon mass $m_{\mathrm{ph}}$ is real. In Fig. 1(a), the T response is computed in the optical range, exhibiting the expected Drudelike behaviour with the characteristic resonance frequency $\omega_{\mathrm{p}}$ around $0.9 \times 10^{15} \mathrm{rad} / \mathrm{s}$. In this case, since $\omega_{\mathrm{p}}$ is independent of $k$ (only the local response is relevant for the transverse component, see equation (30)), then we can readily compute the corresponding photon mass $m_{\mathrm{ph}}$ using (1) and (32), resulting in $m_{\mathrm{ph}}=1.054 \times 10^{-36} \mathrm{~kg}$. This value is several orders of magnitude smaller than the electron mass and is roughly within the same numerical range reported in media such as $\mathrm{SiO}_{2}$-glass using methods completely different from ours, e.g., see [31]. The corresponding photon mass length scale $\lambda_{\mathrm{ph}}$ is computed using (2), giving $\lambda_{\mathrm{ph}}=2.09 \mu \mathrm{m}$, also within the optical wavelength scale.

Fig. 1(b) shows the longitudinal response function for various values of the resonance frequency $\omega_{\mathrm{p}}$, which is wavenumber-dependent, in contrast to the $T$ response case of the top figure. As can be seen from the graph in Fig. 1(b), for each fixed value of $k$, a given Proca MTM exhibits a Drudelike response frequency function qualitatively similar to the $\mathrm{T}$ response case (Fig. 1(a)). However, changes in the effective wavelength-structure of the applied field alters $k$ via $k=2 \pi / \lambda$ and hence may considerably modify the resonance structure of the $L$ response. We should note however that according to (32), the resonance frequency $\omega_{\mathrm{p}}$ can be modified by either changes in the applied field wavelength $\lambda$ or by altering the value of the photon mass $m$ (by redesigning the MTM). This last point will be taken up again in Sec. V-B.

We further note that because for propagating Proca waves with real photon mass the condition $\omega>m c$ holds, it follows

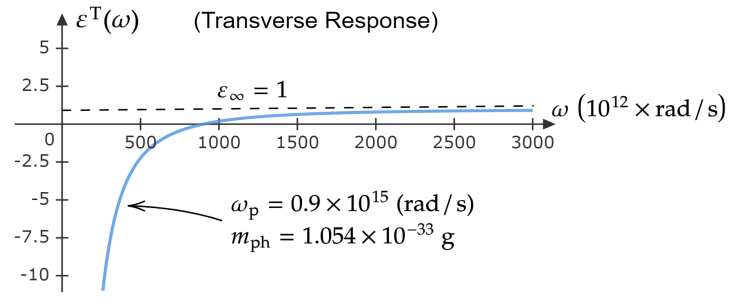

(a)

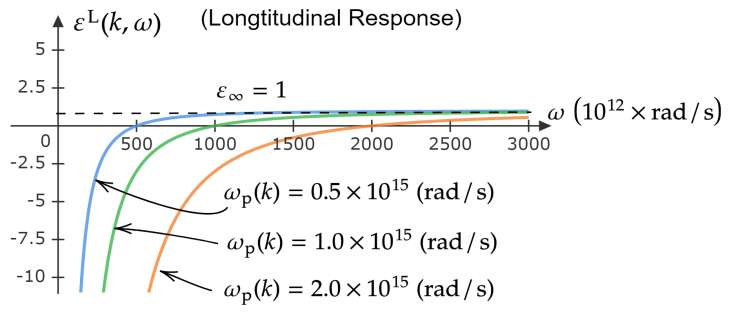

(b)

Fig. 1. The optical transverse (T) and longitudinal (L) dielectric function responses of the Proca MTM as function of frequency $\omega$ with real photon mass. (a) $\mathrm{T}$ response case for a fixed photon mass. (b) $\mathrm{L}$ response case for varying $k$ and/or $m$, leading to changes in the resonance frequency $\omega_{\mathrm{p}}$ that are dependent on the wavelength-structure of the applied field.

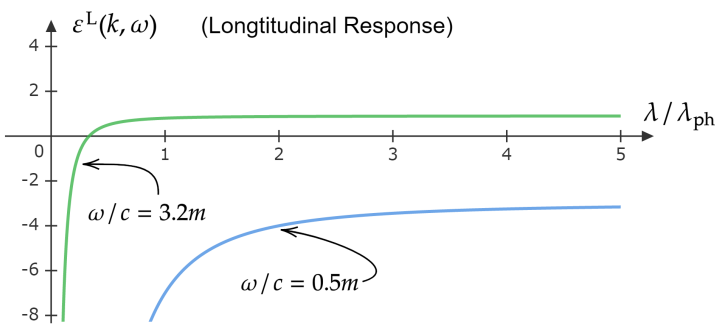

Fig. 2. The dependence of the L dielectric function of a Proca MTM with real photon mass on the wavelength $\lambda$ of the external field. The results are shown for two representative cases of fixed frequency $\omega$. The field wavelength is normalized with respect to the photon mass wavelength $\lambda_{\mathrm{ph}}$.

from (30) that $\varepsilon^{\mathrm{T}}(\omega)>0$ whenever the Proca wave is excited, while $\varepsilon^{\mathrm{T}}(\omega)$ is negative only inside the stopband band, see Fig. 1(a). On the other hand, the behaviour of $\varepsilon^{\mathrm{L}}(k, \omega)$ described by (31) is somehow more complicated. We know that $\varepsilon^{\mathrm{L}}(k, \omega)=0$ whenever there is a Proca wave propagation. Simple calculations reveal that $k>(1 / c)\left(\omega^{2}-m^{2} c^{2}\right)^{1 / 2}$ implies $\varepsilon^{\mathrm{L}}(k, \omega)<0$. The longitudinal dielectric function $\varepsilon^{\mathrm{L}}(k, \omega)$ is therefore negative whenever this condition holds, and that particularly always happens in the short wavelength limit $k \rightarrow \infty$ as will be illustrated below.

The dependence of the $\mathrm{L}$ response dielectric function on $k=2 \pi / \lambda$ is easily computed as

$$
\varepsilon^{\mathrm{L}}(k, \omega)=1-\left(\frac{m c}{\omega}\right)^{2}\left[1+\left(\frac{\lambda_{\mathrm{ph}}}{\lambda}\right)^{2}\right] .
$$

This is illustrated in Fig. 2 where $\varepsilon^{\mathrm{L}}(k, \omega)$ is tabulated versus the excitation wavelength $\lambda$ for two fixed frequencies. It is easy to show that the long-wavelength limit $\lambda \rightarrow \infty(k \rightarrow 0)$ 


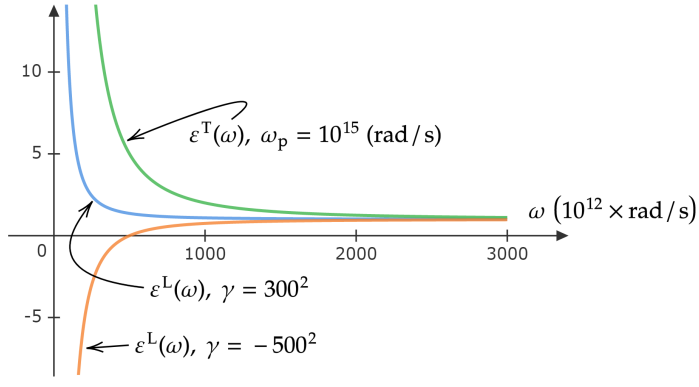

Fig. 3. The $\mathrm{T}$ and $\mathrm{L}$ response functions for imaginary photon mass. Here, basic photon mass quantities such as $m=\mathrm{i}|m|, k=\mathrm{i}|k|$, and $\lambda_{\mathrm{ph}}=\mathrm{i}\left|\lambda_{\mathrm{ph}}\right|$ have all become imaginary. The $\mathrm{T}$ response $\varepsilon^{\mathrm{T}}$ is always positive. On the other hand, for the $\mathrm{L}$ response, the qualitative form of the behaviour of $\varepsilon^{\mathrm{L}}$ depends on the sign of the dimensionless parameter $\gamma=\left[1-\left(\lambda_{\mathrm{ph}} / \lambda\right)^{2}\right]$.

is given by

$$
\lim _{\lambda \rightarrow \infty} \varepsilon^{\mathrm{L}}(k, \omega)=1-\left(\frac{m c}{\omega}\right)^{2} .
$$

Depending on the value of the ratio $m c / \omega$, this limit can be either positive or negative. In Fig. 2 we show two possible cases corresponding to $\omega=0.5 m c$ and $\omega=3.2 m c$. If we choose a value for the photon mass equal to the one in Fig. 1 (a), then these two cases correspond to frequencies given by $\omega=4.5 \times 10^{14} \mathrm{rad} / \mathrm{s}$ and $\omega=2.9 \times 10^{15} \mathrm{rad} / \mathrm{s}$, respectively. The long-wavelength behaviour encapsulated by (36) demonstrates that for Proca MTM, spatial dispersion (nonlocal) effects would cease to be important when the following condition is not satisfied:

$$
\lambda \ll \lambda_{\text {ph }} .
$$

In general, we already know that every nonlocal material/metamaterial has some characteristic spatial scale such that any excitation field with wavelength beyond this scale will not lead to appreciable nonlocal response in medium [48], [51], [71]. It appears then that this critical spatial scale is captured by $\lambda_{\mathrm{ph}}$, the photon mass wavelength defined by (2). ${ }^{6}$ This explains the physical significance of the new parameter $\lambda_{\mathrm{ph}}$ introduced by (54). Indeed, in Proca metamaterials, nonlocality (present only in the L dielectric function) becomes important only for short-wavelength components with $\lambda<\lambda_{\mathrm{ph}}$. Therefore $\lambda_{\mathrm{ph}}$ plays the role of a nonlocality length scale in which all fields components with shorter wavelength content experience spatial dispersion (similar to the role played by the lattice period in periodic structures such as crystals [50], [66], various mainstream MTMs, and the Deybe length in plasma [52].) For example, if we use the photon mass of Fig. 1(a), then this critical spatial scale was found above to be around roughly $\lambda_{\mathrm{ph}} \approx 2 \mu \mathrm{m}$. Incident light with wavelength significantly larger than $2 \mu \mathrm{m}$ will then not experience strong nonlocal effects and the $\mathrm{L}$ response function becomes in that case similar to the $\mathrm{T}$ response Drude-like form.

Finally, let us briefly consider what happens when the photon mass is imaginary, i.e., when $m^{2}<0$. In this case, the $\mathrm{T}$ dielectric function will fail to exhibit the Drude-like behaviour encountered previously. For the L response, the

${ }^{6}$ This point will be further explored in Sec. V-A. situation is slightly more complicated. To see this let us explicitly compute $\varepsilon^{\mathrm{L}}$ for imaginary photon mass $m=\mathrm{i}|m|$. Using (35), this gives:

$$
\varepsilon^{\mathrm{L}}(k, \omega)=1+\frac{|m|^{2} c^{2}}{\omega^{2}}\left[1-\frac{\left|\lambda_{\mathrm{ph}}\right|^{2}}{\lambda^{2}}\right],
$$

where we note that $|m|$ and $\left|\lambda_{\mathrm{ph}}\right|$ are both positive real numbers. ${ }^{7}$ We have then two distinct cases: 1) when $\lambda>\lambda_{\text {ph }}$, we recover the Drude-like model of the real mass scenario. 2) when $\lambda<\lambda_{\text {ph }}$ a sign reversal is introduced. These three possibilities for the $\mathrm{T}$ and $\mathrm{L}$ response functions are collectively illustrated in Fig. 3. As we can see, the $\mathrm{T}$ function $\varepsilon^{\mathrm{T}}$ is no longer Drude-like for imaginary photon mass. On the other hand, the $\mathrm{L}$ response $\varepsilon^{\mathrm{L}}$ can assume a Drude-like behaviour depending on the value of the applied field wavelength or wavenumber.

\section{Proca Waves}

Given that the Proca MTM's nonlocal dielectric tensor has been determined and decomposed into its respective $\mathrm{T}$ and $\mathrm{L}$ wave parts, the natural step now is to compute the dispersion relations of the modes that may propagate through this material domain with proper excitation. Since the Proca MTM is isotropic, we expect to work with both $\mathrm{T}$ and $\mathrm{L}$ waves when the medium is properly excited. The well-known dispersion relations for these $\mathrm{T}$ and $\mathrm{L}$ modes, respectively, are the following [50], [67]

$$
\varepsilon^{\mathrm{T}}(k, \omega)-n^{2}=0, \quad \varepsilon^{\mathrm{L}}(k, \omega)=0,
$$

where the index of refraction $n$ is defined by

$$
n^{2}:=\frac{k^{2} c^{2}}{\omega^{2}} .
$$

Using (30) and (31) in (39), we arrive at

$$
\omega^{2}=c^{2} k^{2}+c^{2} m^{2}=c^{2} k^{2}+\frac{m_{\mathrm{ph}}^{2} c^{4}}{h^{2}},
$$

which is the same dispersion relation for both the transverse and longitudinal waves.

Furthermore, with the help of (1), $E_{\mathbf{p}}=\hbar \omega$, and $\mathbf{p}=\hbar \mathbf{k}$, the dispersion relation (41) then becomes

$$
E_{\mathbf{p}}^{2}=c^{2}|\mathbf{p}|^{2}+E_{\mathrm{ph}}^{2},
$$

where

$$
E_{\mathrm{ph}}:=m_{\mathrm{ph}} c^{2}
$$

is defined as the massive photon's mass energy obtained by mean of the traditional material particle's energy $E=m c^{2}$ when its mass $m$ is replaced by the photon mass $m_{\mathrm{ph}}$. For conventional (massless) electromagnetism, this energy $E_{\mathrm{ph}}$ is identically zero. We also introduce the photon mass frequency $\omega_{\text {ph }}$ defined by

$$
\omega_{\mathrm{ph}}:=\frac{E_{\mathrm{ph}}}{\hbar}=\frac{m_{\mathrm{ph}} c^{2}}{\hbar}=m c .
$$

The frequency $\omega_{\text {ph }}$ may be interpreted as the "particle internal clock" or the frequency of the quantum wave corresponding to massive photon's mass energy $E_{\mathrm{ph}}$ [72], [73].

\footnotetext{
${ }^{7}$ When $m$ is imaginary, the photon mass wavelength is imaginary and is given by $\lambda_{\mathrm{ph}}=\mathrm{i}\left|\lambda_{\mathrm{ph}}\right|$.
} 


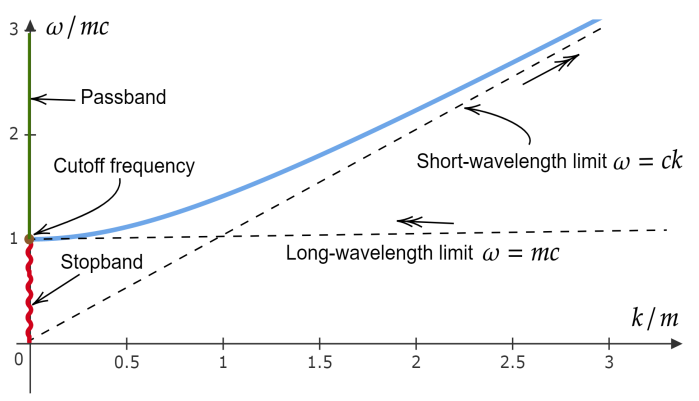

Fig. 4. The dispersion relation of the Proca wave with real normalized mass parameter $m$. Note that the dimension of $m$ is $1 / \mathrm{m}$ so the normalized frequency $\omega / m c$ and normalized wavenumber $k / m$ are both dimensionless.

Because of the importance of this result and for future references, we collect the above conclusions in the following theorem:

Theorem IV.1. Both of the longitudinal and transverse Proca waves are governed by the same dispersion relation given by (41) for the classical version and (42) for the quantum form.

It is remarkable that (42) is exactly the same as the relativistic massive particle dispersion (10) but this time obtained via the Proca MTM dispersion relation (39), providing another physical confirmation of the Proca MTM dielectric tensor (28). In other words, the temporal and spatial dispersion profiles of the Proca MTM (29) already contain the full relativistic structure of the dispersion relation (10). This is why one may also call the Proca medium a kind of "relativistic metamateials" although we will stick with the terminology of Proca MTM here.

The dispersion relation (41) is plotted in Fig. 4, where we also highlight the asymptotic short- and long-wavelength limits $\omega=c k$ and $\omega=m c$, respectively. In particular, note that the short-wavelength limit $\omega=c k$ corresponds to the conventional dispersion relation of massless photons. physically, this can be explained by the fact that photons with very large $k$ also have very large frequency and hence are highly energetic. It is well known that particles with very large energy experiences diminishing inertial effects, the so-called ultra-relativistic limit $[46] .^{8}$

We also observe that a cutoff frequency exists for Proca waves with real mass, which coincides with the longwavelength limit $\omega=m c$. The existence of cutoff frequency can be most directly proved by solving for the wavenumber $k$ in (42) to find

$$
k(\omega)=\frac{1}{c} \sqrt{\omega^{2}-\omega_{\mathrm{ph}}^{2}}=\frac{1}{c} \sqrt{\omega^{2}-m_{\mathrm{ph}}^{2} c^{4} / \hbar^{2}} .
$$

For pure propagating modes, $k$ must be real, leading to the excitation condition $\omega>\omega_{\text {ph }}$ with cutoff frequency $\omega_{c}=$

\footnotetext{
${ }^{8}$ For example, in the Lorentz model of the dielectric function, electron mass effects vanishes at the high-frequency limit $\omega \rightarrow \infty$ as a simple analysis based on Newtonian dynamics can quickly reveal, e.g., see [67], [68], [71]. In the relativistic field theory framework [6], [71], the standard explanation is that the energy of the particle (photon) becomes very large compared with the restmass energy $m_{\mathrm{ph}} c^{2}$, i.e., $\hbar \omega \gg m_{\mathrm{ph}} c^{2}$ so in this case massive photons at the infinite-frequency-wavenumber limit behave essentially as massless particles.
}

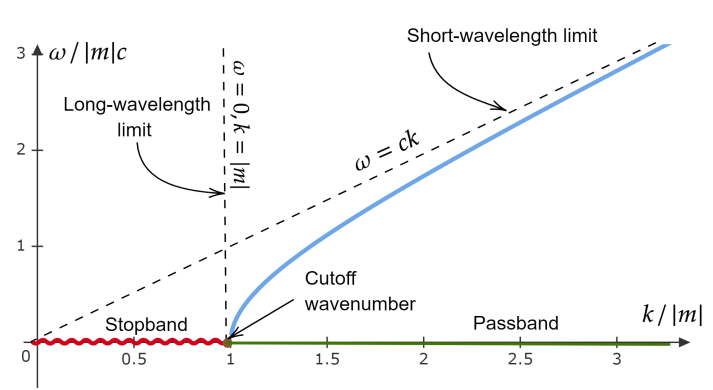

Fig. 5. The dispersion relation of the Proca wave with imaginary normalized mass parameter $m=\mathrm{i}|m|$. The normalized frequency $\omega /|m| c$ and normalized wavenumber $k /|m|$ are both dimensionless.

$\omega_{\text {ph }}$. That is, the photon mass frequency given by (44) is the minimum frequency below which massive photons or Proca waves cannot be effectively launched into the Proca MTM domain. This cutoff is directly proportional to the photon mass $m_{\mathrm{ph}}$. Indeed, for $\omega<\omega_{\mathrm{ph}}, k(\omega)$ becomes pure imaginary, leading to evanescent waves. While the latter modes are still valid solutions for Maxwell's equations in Proca MTMs, their quantization does not lead to "real" photons (whether massive or massless) and thus are not considered underlying waves for effective power transfer processes.

However, it is interesting to note that the derivation of the cutoff condition $\omega>\omega_{\mathrm{ph}}$ depended on $\omega_{\mathrm{ph}}$ being real. For real photon mass, this is the case, but when $m_{\text {ph }}$ becomes imaginary, $\omega_{\mathrm{ph}}^{2}$ is negative and (45) reduces to

$$
k(\omega)=\frac{1}{c} \sqrt{\omega^{2}+\left|\omega_{\mathrm{ph}}\right|^{2}}=\frac{1}{c} \sqrt{\omega^{2}+\left|m_{\mathrm{ph}}\right|^{2} c^{4} / \hbar^{2}},
$$

which is the correct dispersion relation for imaginary photon mass. The relation (46) implies that no cutoff frequency exists for Proca waves with imaginary photon mass. In other words, if the photon mass is imaginary, then Proca waves will always be excited for any nonzero frequency $\omega>0$. Since it would be easy to test these statements in the lab, the previous analysis provides a method to determine whether the photon mass is real or imaginary for a given nonlocal MTM under investigation.

We depict the dispersion relation for the case of imaginary photon mass in Fig. 5. There is no cutoff frequency here and in general all positive frequencies are allowed. However, there exists a lower bound on $k$, hence a minimum wavenumber $k=|m|$ below which no Proca wave with imaginary photon mass is allowed. Correspondingly, an upper bound on the wavelength $\lambda_{\max }=2 \pi /|m|$ exists, which is essentially the photon mass wavelength $\left|\lambda_{\text {ph }}\right|$ introduced by (2). Therefore, the long-wavelength limit is different from the case of real photon mass illustrated in Fig. 4 where the latter was $k \rightarrow 0$ but should now be replaced by $k \rightarrow k_{\min }=|m|$. Nevertheless, the short-wavelength limit $k \rightarrow \infty$ is the same in both the real and imaginary photon mass scenarios. There also exists a wavelength or wavenumber stop- and pass-bands associated with the ranges $0<k<|m|$ and $|m|<k<\infty$, respectively. In this special case, any Proca wave with wavelength $\lambda$ satisfying $\lambda>\left|\lambda_{\mathrm{ph}}\right|=2 \pi /|m|$ would not be excited. 


\section{Comparison Between Proca Waves and Conventional Waves}

From the classical viewpoint, one of the most important feature enjoyed by Proca waves is the inseparability of the $\mathrm{T}$ and $\mathrm{L}$ polarization components entering into the field composition of these waves. It might be tempting to think of the Proca wave as nothing but two degenerate $\mathrm{T}$ and $\mathrm{L}$ waves sharing the same eigenvalues (frequencies) specified by (42). However, we believe this interpretation is not the most appropriate one. Instead, we suggest that Proca waves should be viewed as essentially exemplifying a new genre of electromagnetic wave modes, a kind of "relativistic wave" not simply reducible to conventional $\mathrm{T}$ or $\mathrm{L}$ modes when each excited independently. The reasons supporting this claim are the following:

1) The $T$ and $L$ modes above have exactly the same dispersion relations though with complementary polarization structures. This is then not another case of repeated roots of a given dispersion relation as typically seen in various degeneracy phenomena in eigenvalue problems.

2) The full canonical quantization of Proca waves (to be addressed by the author in a separate paper), resulting in the standard massive-photon eigenmode expansion, explicitly reveals that the underlying wave has three polarization components and hence strongly suggests that the classical Proca wave is not a superposition of two modes, but essentially one combined mode (Proca mode) that should be treated as a whole in order to retain some continuity between the classical and quantum pictures (one classical wave is leading to one quantum process, i.e., the massive photon, and vice verse).

3) As will be discussed elsewhere in the quantum-theoretic treatment, Proca waves' polarization vectors are most efficiently viewed as 4-vectors living in the 4-dimensional Minkowsky space of SR. In that perspective, there are no separate $\mathrm{L}$ and $\mathrm{T}$ modes, but a single combined $\mathrm{L}$ $\mathrm{T}$ polarization structure generated by a subgroup of the full Lorentz group (the symmetry group of SR).

At a deeper level, we may say that the existence of a larger symmetry group (Lorentz group) in the case of Proca waves confers on the latter an intrinsic unity of structure. Hence, a decomposition of Proca waves into two distinct $\mathrm{L}$ and $\mathrm{T}$ waves becomes relatively superficial and hence unwarranted. The increase in the number of polarization units from 2 to 3 when going from massless photons (conventional Maxwell theory) to Maxwell-Proca electromagnetics is connected with the move from nonrelativistic 3-dimensional geometry to the 4-dimensional Minkowskian geometry of special relativity.

Note that the existence of hybrid waves - where it is not possible to separate $\mathrm{L}$ and $\mathrm{T}$ polarization into distinct modes - is not unique to Proca wave for it can occur in some anisotropic media [47], [50], [67]. What is remarkable though is that Proca MTMs are isotropic media and in such domains conventional Maxwell theory (massless photons) treats the $\mathrm{L}$ and $\mathrm{T}$ modes as two independent modes. One of the main advantages of Proca antennas (to be explored later in Sec. V-C) is the ability to exploit nonlocal antenna technology to engi- neer radiation with three independent degrees of polarization instead of two [54], [55], [74], but now while working with a single (Proca) mode instead of the cumbersome excitation of two different $\mathrm{L}$ and $\mathrm{T}$ modes in order in order to properly combine them as desired.

It is also instructive to examine the various possible types of propagation velocities affiliated to Proca waves and compare them with the quantum particle (massive photon) formula. The phase velocity is defined by $\mathbf{v}_{p}:=\hat{k}(\omega / k)$. Using the dispersion relation (41), this leads to

$$
\mathbf{v}_{p}=\hat{k} c \frac{\sqrt{k^{2}+\left(\omega_{\mathrm{ph}} / c\right)^{2}}}{k}=\hat{k} c \frac{\omega}{\sqrt{\omega^{2}-\omega_{\mathrm{ph}}^{2}}} .
$$

In vacuum, photons are massless or $\omega_{\mathrm{ph}}=0$, hence from (47) $\mathbf{v}_{p}=\hat{k} c$, which is the correct relation for such vacuum waves. The group velocity on the other hand is defined by $\mathbf{v}_{g}:=\nabla_{\mathbf{k}} \omega(\mathbf{k})$. Using (41) again, we find

$$
\mathbf{v}_{g}=\hat{k} c \frac{k}{\sqrt{k^{2}+\left(\omega_{\mathrm{ph}} / c\right)^{2}}}=\hat{k} c \frac{\sqrt{\omega^{2}-\omega_{\mathrm{ph}}^{2}}}{\omega},
$$

Moreover, for massless photons (waves in vacuum), the group velocity reduces to $\mathbf{v}_{g}=\hat{k} c$. Nevertheless, relation $\mathbf{v}_{g} \cdot \mathbf{v}_{p}=c^{2}$ holds for all cases (massless and massive photons alike.)

Fig. 6 gives the group and phase velocities as function of frequency for real photon mass. We note that $v_{p}$ is in general greater than the speed of light while $v_{g}<c$ approaches $c$ asymptotically. The latter behaviour is the foundation of the possibility to incorporate SR with massive electromagnetism [10]. Therefore, it is group velocity that represents the physical speed of photons. A confirmation of this can be found if we solve for $\omega$ in (48) and use the Planck-Einstein relation $E=$ $\hbar \omega$, resulting in

$$
E=\frac{m_{\mathrm{ph}} c^{2}}{\sqrt{1-v_{g}^{2} / c^{2}}} .
$$

The relation (49) is identical to the relativistic energy expression of a particle moving with speed $v_{g}$ and mass $m_{\mathrm{ph}}$ [46], [75]. Therefore, Proca waves do behave like a massive relativistic particle. Since $v_{g} \leq c$ and $\omega>\omega_{\mathrm{ph}}$, photons with real mass possess positive energy and hence are physically allowed. On the other hand, if the photon mass is imaginary, photons with real energy must posses group velocities exceeding $c$ in order to yield real energy [29], and hence such Proca waves are superluminal. ${ }^{9}$ The phase and group velocities of imaginary mass Proca waves is shown in Fig. 7. We note that in contrast to the real-mass case of Fig. 6, the group velocity is greater than $c$ while the phase velocity is always less than $c$.

The natural question now is what material photon particle velocity should be used in comparison to the two Proca wave's velocities introduced above. We propose the following definition of the massive photon's mass velocity:

$$
\mathbf{v}_{\mathrm{ph}}:=\frac{c^{2}}{E_{\mathbf{p}}} \mathbf{p}
$$

\footnotetext{
${ }^{9}$ Superluminal light and microwave signals are experimentally observable in waveguides and dispersive media [76] and even in the near-field zone in vacuum [77]-[80]. For in-depth general view, see [81].
} 


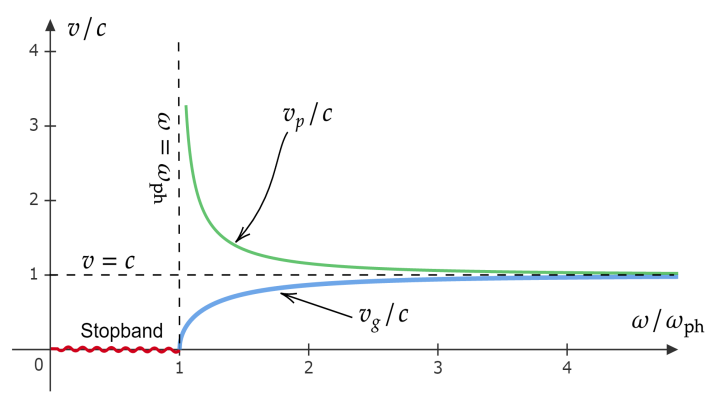

Fig. 6. Phase and group speeds ( $v_{p}$ and $v_{g}$, respectively) for Proca waves with real photon mass. In order for the corresponding Proca wave to be excited, the condition $\omega>\omega_{\mathrm{ph}}$ must be satisfied hence both $v_{p}$ and $v_{g}$ are real only for frequencies above the photon mass $\omega_{\mathrm{ph}}$.

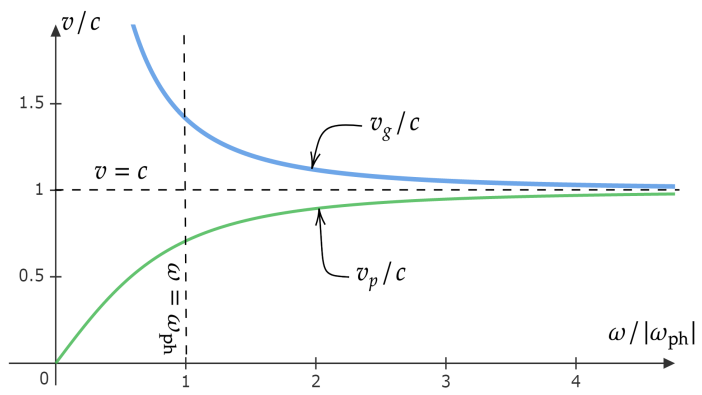

Fig. 7. Phase and group speeds ( $v_{p}$ and $v_{g}$, respectively) for Proca waves with imaginary photon mass. In this case the condition $\omega>\omega_{\mathrm{ph}}$ is not required and both $v_{p}$ and $v_{g}$ are real for any frequency. The photon mass frequency is itself imaginary $\omega_{\mathrm{ph}}=\mathrm{i}\left|\omega_{\mathrm{ph}}\right|$ and the expressions for the phase and group speeds are $v_{p}=c \omega / \sqrt{\omega^{2} /\left|\omega_{\mathrm{ph}}\right|^{2}+1}$ and $v_{g}=c \sqrt{\omega^{2} /\left|\omega_{\mathrm{ph}}\right|^{2}+1} / \omega$, respectively.

which comes from SR [5], [6], [46]. Substituting (42) into (50) immediately gives

$$
\mathbf{v}_{\mathrm{ph}}:=c \frac{\mathbf{p}}{\sqrt{|\mathbf{p}|^{2}+E_{\mathrm{ph}}^{2} / c^{2}}} .
$$

Direct calculation of (50) with the dispersion relation (42) reveals that $\mathbf{v}_{\mathrm{ph}}=\mathbf{v}_{g}$ (relation (53) below). Therefore, we may then substitute (53) and (49) into (50) and after identifying $E_{\mathbf{p}}$ with $E=\hbar \omega$, the following expression for the momentum of the massive photon corresponding to the Proca wave is obtained:

$$
\mathbf{p}=\frac{m_{\mathrm{ph}} \mathbf{v}_{g}}{\sqrt{1-v_{g}^{2} / c^{2}}},
$$

which is the correct relativistic expression of a particle with mass $m_{\mathrm{ph}}$ and velocity $\mathbf{v}_{g}$.

We give two immediate observations on the photon momentum determined by (52). First, It can be shown that our definition of the photon velocity using (50), which led to the momentum expression (52), agrees with the Abraham definition of the photon-in-dielectric momentum. This may shed some light on the (still ongoing) Abraham-Minkowski debate [82], [83], but we do not pursue this issue further here. Second, it is to be noted that in the limit of massless photons $m_{\mathrm{ph}} \rightarrow 0$, the group velocity approaches the speed of light $\left(v_{g} \rightarrow c\right)$ according to (48). Therefore, in this case the energy and momentum given by the expressions (49) and (52) do not vanishes in the limit $m_{\mathrm{ph}} \rightarrow 0$ because they have the form $0 / 0$. The limits actually correspond to the massless photon energy and momentum and these are not zero for any $\omega \neq 0$ frequency.

Finally, since one may question the use of the photon energy $E_{\mathbf{p}}=\hbar \omega$ in (50) instead of the actual photon mass energy $E_{\mathrm{ph}}$ given by (43), so we provide two reasons for our choice:

1) The relation (50) is in fact the correct relativistic velocity formula valid for both massive and massless particles [72]. If we would like the correspondence between the classical case (Proca wave) and the quantum process (massive photon) to be exact, we expect the proper SR formula to apply.

2) Using $E_{\mathrm{ph}}$ in (50) instead of $E_{\mathbf{p}}$ will lead to the nonphysical result of infinite speed when the photon mass $m_{\mathrm{ph}}$ goes to zero. On the other hand, the formula (51) turns out to be exactly the same as the group velocity (48) as can be readily verified by using $\mathbf{p}=\hbar \mathbf{k}$.

Summing up, we have established another remarkable correspondence between Proca waves, i.e., classical EM waves excited in Proca nonlocal MTMs, and massive photons where the following equality holds:

$$
\mathbf{v}_{\mathrm{ph}}(\mathbf{p})=\mathbf{v}_{g}(\mathbf{k}) .
$$

This key relation supplies additional evidence in support of considering Proca waves as essentially a single mode propagating in an isotropic medium (Proca MTM) with three independent polarization units. The relation (53) offers a translation from the quantum particle motion language (massive photons) to classical EM waves (Proca modes), hence illustrating an inherent electromagnetic wave-particle duality.

\section{Metamaterial Design and Applications to PROCA ANTENNAS}

\section{A. General Considerations for the Design of Proca Metama- terials}

While the full design and implementation of an actual physical prototype capable of realizing Proca MTMs is outside the scope of this paper, some general considerations regarding lab actualization may be briefly visited here. In what follows, we analyze the main features of the temporal and spatial dispersion profiles associated with this new genre of MTMs, with focus on existing and potential tradeoffs between various design parameters.

1) On dissipation in Proca MTMs: First, we say few words about losses. For real or imaginary photon mass, the Proca MTM dielectric function profile (28) is hermitian and hence represents a lossless medium. In general, for simplicity in the mathematical analysis we follow the convention of condensedmatter and plasma physics where dispersion analysis is based on the hermitian part of the dielectric function. Small losses can be added as a perturbation and computed using standard methods [48], [51], [52], [66]-[68]; in that case the antihermitian part of the dielectric tensor becomes the carrier of information on dissipation. However, it is straightforward to incorporate even strong losses in the theory of Proca MTMs 
by simply replacing $\pm|m|^{2}$ by $\pm|m|^{2}+\mathrm{i} \zeta, \zeta \in \mathbb{R}^{+}$, where the imaginary part i $\zeta$ describes dissipation. The exact dispersion theory of this case is considerably more complicated and is outside the scope of this paper. Since in most practical applications dissipation is treated as a perturbation added to the exact lossless case [49], [54], [55], [67], the basic theory of massive electromagnetism with pure real or imaginary photon mass outlined above should be adequate as an initial theory of Proca MTMs.

2) An alternative physical interpretation for $\lambda_{\mathrm{ph}}$ : Next, let us revisit the concept of the photon mass wavelength $\lambda_{\mathrm{ph}}$ introduced by (2). The photon mass frequency $\omega_{\mathrm{ph}}$ defined via (44) represents a natural frequency of the massive photon system, a sort of "photon internal clock." It is also possible to view the same quantity from a different perspective. From the definition of the spacetime Fourier transform (12) and (13), the quantity $\mathbf{k}$ represents the spatial frequency of the field and this is proportional to the inverse of the field wavelength. This motivates the introduction of the "photon mass wavenumber" $k_{\mathrm{ph}}$ defined by means of the relation

$$
k_{\mathrm{ph}}:=\frac{2 \pi}{\lambda_{\mathrm{ph}}}=m=\frac{\omega_{\mathrm{ph}}}{c},
$$

where the second equality can be readily verified from (2) while the third follows from (44). Since the dispersion relation of a massless photon is given by $k=\omega / c$, we may physically interpret $\lambda_{\mathrm{ph}}$ as the wavelength of $a$ massless photon with frequency $\omega_{\text {ph }}$ propagating in vacuum. From the condition $\omega>\omega_{\mathrm{ph}}$, this is also the cutoff wavelength of Proca waves (with real photon mass) if they were to propagate in vacuum. The parameter $k_{\mathrm{ph}}$ then provides information about how data on the photon mass can be transformed into data on the corresponding Maxwellian wave in vacuum. To illustrate how this can be done in practice, we next look into some inherent tradeoffs in Proca MTMs design.

3) Fundamental Tradeoffs in the Process of Proca MTM Design: From (54) we can see that the critical nonlocality wavelength $\lambda_{\mathrm{ph}}$ is proportional to the inverse of the photon mass $m_{\mathrm{ph}}$, while $\omega_{\mathrm{ph}}$ is directly proportional to the mass itself. as we have established in Sec. IV-B, the length scale $\lambda_{\mathrm{ph}}$ represents a characteristic nonlocal spatial scale where spatial dispersion must be significant for wavelengths satisfying the condition (37), i.e., $\lambda<\lambda_{\mathrm{ph}}$ or equivalently $k>k_{\mathrm{ph}}$. Consequently, there is a fundamental tradeoff between the temporal and spatial cutoff frequencies in Proca MTMs: The smaller the cutoff frequency $\omega_{\mathrm{ph}}$, the stronger the nonlocality that need to be implemented in the MTM in order to attain a Proca-type MTM response function, and vice versa.

There is then a price to pay for building a Proca MTM at lower frequencies like microwave or terahertz band, and that is the need to engineer media with strong spatial dispersion profiles. This is one of the most important features introduced by the Proca medium as a new generation of MTMs where nonlocality is to be embraced as a positive trait to be exploited in future application; i.e., nonlocality should not be treated as a "bug" to be minimized or removed in the design and development processes of complex media.

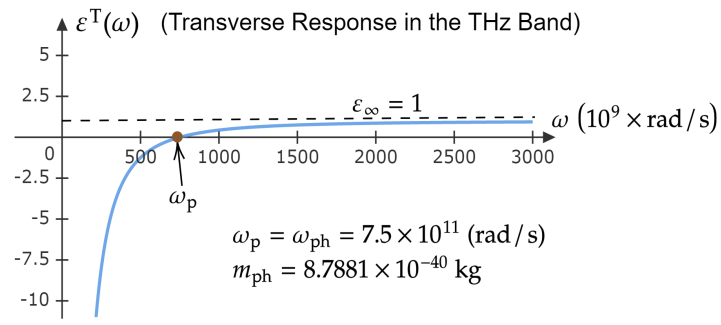

Fig. 8. A possible terahertz $(\mathrm{THz})$ realization of the transverse $(\mathrm{T})$ dielectric function response of the Proca MTM with real photon mass. The resonance frequency $\omega_{\mathrm{p}}(k \rightarrow 0)=\omega_{\mathrm{ph}}=m c$ can be used to estimate the required value of the photon mass $m_{\mathrm{ph}}$ through the relation $m_{\mathrm{ph}}=\omega_{\mathrm{ph}} \hbar / c^{2}$.

\section{B. Basic Material Design Examples}

A design example is now given to illustrate these general conclusions. For simplicity, we focus on the real mass case where Proca waves are excited only when $\omega>\omega_{\mathrm{ph}}$. Let us consider the realization of a Proca MTM operating in the terahertz $(\mathrm{THz})$ band. The design of the transverse dielectric profile is shown in Fig. 8 where we choose the MTM resonance frequency to be $\omega_{\mathrm{p}}=7.5 \times 10^{11} \mathrm{rad} / \mathrm{s}$. The corresponding photon mass can be readily computed as explained in the caption, yielding a value around $m_{\mathrm{ph}}=8.8 \times 10^{-40} \mathrm{~kg}$. Clearly this is three orders of magnitude smaller than the corresponding value found for realizing the medium in the optical range (Fig. 1). On the other hand, the corresponding value for $\lambda_{\mathrm{ph}}$ is $0.25 \mathrm{~mm}$. Physically, this means that a Maxwellian EM wave in vacuum would have to have a wavelength of order $0.25 \mathrm{~mm}$ at the frequency at which Proca waves can be excited in the Proca MTM. Therefore, propagation of Proca waves in Proca MTMs is akin to the propagation of the mmWaves in vacuum. The second physical explanation of $\lambda_{\mathrm{ph}}$, i.e., as a characteristic length scale of nonlocality, implies that the Proca MTM should support nonlocal behaviour up to $0.25 \mathrm{~mm}$. This should be compared with the value we obtained in the optical Proca MTM of Fig. 1 where in that case we found that $\lambda_{\text {ph }}=2 \mu \mathrm{m}$. Therefore, lowering the Proca MTM's resonance frequency $\omega_{\mathrm{p}}$ from $0.9 \times 10^{15} \mathrm{rad} / \mathrm{s}$ in the optical case to $7.5 \times 10^{11} \mathrm{rad} / \mathrm{s}$ in the mmWave range requires an increase in the nonlocality length scale of the MTM by a factor of 150 . Realizing Proca MTMs at lower frequencies then is technologically challenging though improvement is expected in the near future. This is one of the fundamental tradeoffs mentioned in Sec. V-A3.

Indeed, the situation with the longitudinal dielectric function design is considerably more complicated than the transverse case due to the need to establish a strong nonlocal effect in this component of the dielectric tensor. As can be seen from (32), the MTM's resonance frequency $\omega_{\mathrm{p}}$ is a function of $k$ taking the form $\omega_{\mathrm{p}} / \omega_{\mathrm{ph}}=\sqrt{1+\left(k / k_{\mathrm{ph}}\right)^{2}}$. Consequently, we cannot in general find a unique single value for $\omega_{\mathrm{p}}$ because a generic excitation field contains a wide range of wavelength components indexed by $0<k=2 \pi / \lambda<\infty$. It is possible however to outline a simple design procedure to deal with this problem.

We start from the observation that the nonlocal $\mathrm{L}$ profile (31) has only a quadratic term in $k$. This is an example of 
what is called non-resonant type nonlocal MTM [49], [54]. In general, it is known that nonlocal MTMs of this genre contain higher-order terms in $k$. Practically speaking, as $k$ increases, one must include higher powers in $k$ to correctly model the physics of interactions at multiple spatial scales [48], [51]. Thus, for a practical realization of a Proca MTM with the profile (31), one must work only with a limited wavelength components range, that is, we have the restrictions:

$$
k_{\min }<k<k_{\max }, \lambda_{\min }<\lambda<\lambda_{\max },
$$

where $k_{\min / \max }=2 \pi / \lambda_{\max / \max }$. The meaning of the restriction (55) is as follows. The upper bound on the wavelength $\lambda_{\max }$ comes from the condition (37). On the other hand, the lower bound ensures that within such wavenumber/wavelength range, the physical nonlocal dielectric profile of a practical medium to be used for the realization of the Proca MTM (31) has only terms quadratic in $k$ and no higher powers. Examples of such nonlocal media include plasma domains [52], [67] and some periodic structures such as crystals [50], [66].

The corresponding situation with the resonance frequency is illustrated in Fig. 9. Only within the highlighted $k$-axis "nonlocality range," i.e., according to (55), should we seek the operating resonance frequency (design) point of the Proca MTM. The desired such resonance frequency is denoted by $\omega_{\mathrm{p}}^{\star}$. In general, we need to find the value of the photon mass $m_{\mathrm{ph}}$ such that the $\omega_{\mathrm{p}}(k)$ curve will intersect the horizontal line $\omega_{\mathrm{p}}=\omega_{\mathrm{p}}^{\star}$ within the allowable nonlocality range. As can be seen from the Fig. 9, this occurs at some wavenumber $k=k^{\star} \in\left[k_{\min }, k_{\max }\right]$. Parallel to this interval is the frequency range $\left[\omega_{\mathrm{p}, \min }, \omega_{\mathrm{p}, \max }\right] \ni \omega_{\mathrm{p}}$, which represents the "detuning" interval of the resonant frequency. That is, because of the inherent variation in $k$ due to the nonlocality of $\varepsilon^{\mathrm{L}}$, there is an intrinsic fluctuation in the value of the resonance frequency $\omega_{\mathrm{p}}$. The choice of optimum or design point $\left(k^{\star}, \omega_{\mathrm{p}}^{\star}\right)$ depends on minimizing the detuning size measured by $\Delta \omega_{\mathrm{p}}$. Put differently, we would like to chose an operating point such that:

1) The resonance frequency $\omega_{p}$ lies within the desired range of the application at hand (e.g., microwave, mmWave, optical, etc).

2) The fluctuations (detuning) of $\omega_{p}$ is not large enough to cause the resonance frequency to move outside the desired application range mentioned above.

3) The nonlocality range of the practical nonlocal MTM at hand is respected so $k^{\star} \in\left[k_{\min }, k_{\max }\right]$.

Once a decision is made, the value of the photon mass $m_{\mathrm{ph}}$ can be easily obtained from the design point $\left(k^{\star}, \omega_{\mathrm{p}}^{\star}\right) \cdot{ }^{10}$ A possible choice for $k^{\star}$ is the wavenumber/wavelength at which the excitation field is maximal. If this information is not available, then we can use a suitable averaging scheme. In Table IV we list some of these methods with their formulas.

We give a concrete example to illustrate the general design procedure above. We wish to design a Proca MTM with a longitudinal resonance frequency $\omega_{\mathrm{p}}$ and $\lambda_{\max }=0.3 \mathrm{~mm}$ and $\lambda_{\min }=0.1 \mathrm{~mm}$. We estimate the $k^{\star}$ using the arithmetic mean of the wavelength (Table IV), resulting in $k^{\star}=0.5 \times 2 \pi \times 10^{3} \times$

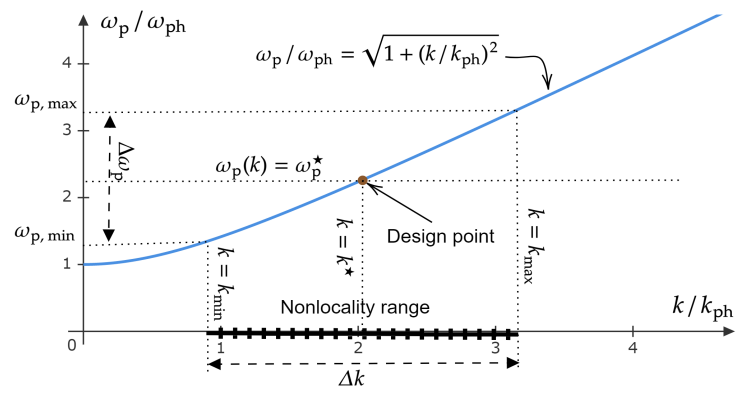

Fig. 9. The general dependence of the longitudinal Proca MTM resonance frequency $\omega_{\mathrm{p}}$ on the wavenumber $k=2 \pi / \lambda$ for real photon mass parameter $m$. The massive photon wavenumber $k_{\mathrm{ph}}=2 \pi / \lambda_{\mathrm{ph}}=m$ and the photon mass frequency $\omega_{\mathrm{ph}}=m c$ are the main material parameters, both reducible to $m_{\mathrm{ph}}$.

\begin{tabular}{cc}
\hline Expression & \multicolumn{1}{c}{ Method } \\
\hline$k^{\star}=\left(k_{\min }+k_{\max }\right) / 2$ & Arithmetic average of $k$ \\
$k^{\star}=\left(k_{\min } k_{\max }\right)^{1 / 2}$ & Geometric average of $k$ \\
$k^{\star}=4 \pi\left(k_{\min }+k_{\max }\right)^{-1}$ & Arithmetic average of $\lambda$ \\
$k^{\star}=2 \pi\left(k_{\min } k_{\max }\right)^{-1 / 2}$ & Geometric average of $\lambda$ \\
$k^{\star}=\max _{k \in \mathbb{R}+|\mathbf{E}(\mathbf{k}, \omega)|}$ & Maximum field strength
\end{tabular}

TABLE IV

LIST OF POSSIBLE METHODS TO SELECT THE VALUE OF THE DESIGN POINT $\left(k^{\star}, \omega_{\mathrm{p}}^{\star}\right)$ IN THE $k-\omega$ DIAGRAM.

$(1 / 0.3+1 / 0.1)=4.1 \times 10^{4} \mathrm{~m}^{-1}$. From the relation $\omega_{\mathrm{p}} / \omega_{\mathrm{ph}}=$ $\sqrt{1+\left(k / k_{\mathrm{ph}}\right)^{2}}$ we solve for $m$ to obtain $\omega_{\mathrm{p}}=c \sqrt{m^{2}+k^{\star 2}}$. The normalized mass is best estimated by setting $\lambda_{\max }=\lambda_{\mathrm{ph}}$ as a direct way to satisfy (37). This immediately gives $m=$ $10^{3} \times 2 \pi / 0.3=2.1 \times 10^{4} 1 / \mathrm{m}$. Therefore, from the above value obtained for $k^{\star}$, we compute $\omega_{\mathrm{p}}=8.8 \times 10^{12} \mathrm{rad} / \mathrm{s}$. It is worth reminding that for real mass $m$, only resonance frequencies satisfying $\omega_{\mathrm{p}}>k^{\star} c=\omega_{\mathrm{ph}}=6.28 \times 10^{12} \mathrm{rad} / \mathrm{s}$ are possible. Otherwise, we must work with imaginary photon mass. Our example then belongs to the former scenario. The final design parameters we obtained are summarized in Table V.

\begin{tabular}{ll}
\hline MTM Quantity & \multicolumn{1}{c}{ Value } \\
\hline$\lambda_{\mathrm{ph}}$ & $0.3 \mathrm{~mm}$ \\
$\lambda_{\min }$ & $0.1 \mathrm{~mm}$ \\
$\lambda_{\max }$ & $0.3 \mathrm{~mm}$ \\
$m_{\mathrm{ph}}$ & $7.3 \times 10^{-39} \mathrm{~kg}$ \\
$k_{\mathrm{ph}}$ & $4.1 \times 10^{4} \mathrm{~m}^{-1}$ \\
$\omega_{\mathrm{ph}}$ & $6.3 \times 10^{12} \mathrm{rad} / \mathrm{s}$ \\
$\omega_{\mathrm{p}}$ & $8.8 \times 10^{12} \mathrm{rad} / \mathrm{s}$ \\
\hline
\end{tabular}

TABLE V

SumMary of A MMWAVE LONGITUdinAl PROCA MTM PROFILE DESIGN EXAMPLE.

${ }^{10}$ See Table II. 


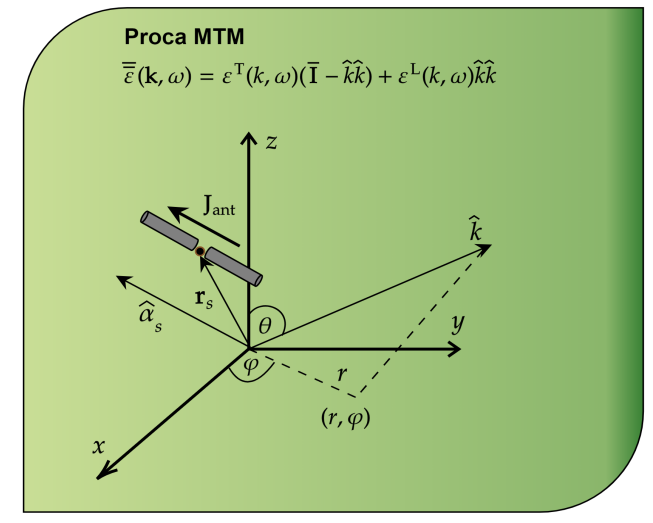

Fig. 10. The nonlocal Proca antenna system. The $\mathrm{T}$ and $\mathrm{L}$ dielectric functions $\overline{\bar{\varepsilon}}^{\mathrm{T}}(k, \omega)$ and $\overline{\bar{\varepsilon}}^{\mathrm{L}}(k, \omega)$ are given by (30) and (31).

\section{Applications: Classical Proca Antennas}

In this Section, we present an application of Proca metamaterials in the field of antenna theory. The presentation is restricted to classical fields, while the subject of quantum Proca antennas will be addressed in a separate paper. For complete details on the background of nonlocal antenna theory, see [54], [55], [74].

We consider a small dipole embedded into the Proca MTM as shown in Fig. 10. The dipole is small in the sense that i) its length $L$ is electrically small, i.e., $L / \lambda \ll 1$, and ii) the ratio between the radius of the dipole cylinder $a$ and $L$ satisfies $a / L \ll 1$. Under these conditions, we may model a time-harmonic dipole antenna by the current distribution [84], [85]:

$$
\begin{aligned}
\mathbf{J}_{\text {ant }}(\mathbf{r}, t) & =\hat{\alpha}_{s} J_{s} \delta\left(\mathbf{r}-\mathbf{r}_{s}\right) e^{-\mathrm{i} \omega_{s} t}, \\
\mathbf{J}_{\text {ant }}(\mathbf{k}, \omega) & =\hat{\alpha}_{s} e^{\mathrm{ik} \cdot \mathbf{r}_{s}} 2 \pi J_{s} \delta\left(\omega-\omega_{s}\right),
\end{aligned}
$$

where $\delta$ stands for the 3-dimensional Dirac delta function. Here, $\mathbf{r}_{s}$ is the location of the dipole midpoint (center); the direction of the dipole is specified by the unit vector $\hat{\alpha}_{s}$; the sinusoidal excitation (circular) frequency is $\omega_{s}$; finally, the frequency-dependent complex-valued quantity $J_{s}=J_{s}\left(\omega_{s}\right)$ measures the excitation current distribution strength. The infinitesimal dipole model (IDM) based on this EM point-source [86] has numerous applications in applied electromagnetics since it can be used to model complex unknown source regions based on arrangement of few dipoles [87]-[101]. For that reason, we focus in what follows on such simple but important antenna type.

The fundamental momentum space quantity needed in order to compute the nonlocal antenna radiation power pattern is $U_{l}(\mathbf{k})$ introduced in [74], which is defined as the density of energy transferred from the source current $\mathbf{J}_{\text {ant }}(\mathbf{r}, t)$ into the $l$ th radiation mode's field per the momentum-space differential volume element $\mathrm{d}^{3} \mathbf{k} /(2 \pi)^{3}$, with its general expression given by (65) in Appendix below B. To compute the radiation power density, the approach adopted in [55] was to multiply the source by a window function with duration $T$ then take the limit when $T \rightarrow \infty$, yielding the momentum-space power spectral density defined by

$$
P_{l}(\mathbf{k}):=\lim _{T \rightarrow \infty} \frac{U_{l}(\mathbf{k})}{T} .
$$

Assuming that a gated version of sinusoidal excitation (56) with duration $T$ is applied, substituting the expression into the $\mathrm{T}$ and $\mathrm{L}$ radiation energy density formulas (80) and (81) in Appendix B, making use of (66), the following radiation power formulas for the Proca infinitesimal dipole are obtained:

$$
\begin{aligned}
& P^{\mathrm{T}}(\varphi, \theta ; \omega)=\frac{\omega\left[(\omega / c)^{2}-m^{2}\right]^{1 / 2}}{2 \varepsilon_{0}(2 \pi)^{3} c^{2}} 2 \pi J_{0} \delta\left(\omega-\omega_{s}\right) \\
& \quad \times\left|(\hat{x} \cos \varphi \sin \theta+\hat{y} \cos \varphi \sin \theta+\hat{z} \cos \theta) \times \hat{\alpha}_{s}\right|^{2}, \\
& P^{\mathrm{L}}(\varphi, \theta ; \omega)=\frac{\omega\left[(\omega / c)^{2}-m^{2}\right]^{1 / 2}}{2 \varepsilon_{0}(2 \pi)^{3} c^{2}} 2 \pi J_{0} \delta\left(\omega-\omega_{s}\right) \\
& \quad \times\left|(\hat{x} \cos \varphi \sin \theta+\hat{y} \cos \varphi \sin \theta+\hat{z} \cos \theta) \cdot \hat{\alpha}_{s}\right|^{2},
\end{aligned}
$$

for the $\mathrm{T}$ and $\mathrm{L}$ Proca antenna radiation power patterns, respectively. The limit (57) was computed with the help of the identity $\left[2 \pi \delta\left(\omega-\omega_{s}\right)\right]^{2}=T 2 \pi \delta\left(\omega-\omega_{s}\right)$ [102] in order to eliminate the arbitrary pulse duration $T$ from the final answer. $^{11}$

In practical applications such as wireless communications and wireless power transfer we would be interested in estimating the total radiated power along a certain angular beam $\Omega_{r}:=\left\{\theta_{1}<\theta<\theta_{2}, \varphi_{1}<\varphi<\varphi_{2}\right\}$. It can be shown that this may be computed by means of the formula [55]

$$
P_{\operatorname{rad}}^{\mathrm{T} / \mathrm{L}}\left(\Omega_{r}\right)=\int_{0}^{\infty} \mathrm{d} \omega \int_{\Omega_{r}} \mathrm{~d} \Omega P^{\mathrm{T} / \mathrm{L}}(\theta, \varphi ; \omega),
$$

which will be used below.

Both the $\mathrm{T}$ and $\mathrm{L}$ radiation power patterns have the same frequency dependence. This is a a characteristic feature of Proca antennas. It is a direct outcome of Theorems B.1 and IV.1. In contrast, the general theory of non-Proca nonlocal MTMs developed in [54], [55], [74] predicts different radiation patterns for the $\mathrm{T}$ and $\mathrm{L}$ wave components, e.g., see some generic examples in [55].

If we add the $\mathrm{T}$ and $\mathrm{L}$ radiation patterns, the resulted total power pattern of a Proca infinitesimal dipole becomes isotopic. To see this, consider the simple example of a dipole located at the origin and oriented along the $z$-axis, i.e., we choose $\hat{\alpha}_{s}=\hat{z}$ and $\mathbf{r}_{s}=0$. Indeed, in such case we may use (60) to integrate only over frequency $\omega$ while keeping the angular dependence, yielding:

$$
\begin{aligned}
& P_{\text {rad }}^{\mathrm{T}}\left(\varphi, \theta ; \omega_{s}\right)=J_{0} \frac{\omega_{s}\left[\left(\omega_{s} / c\right)^{2}-m^{2}\right]^{1 / 2}}{8 \varepsilon_{0} \pi^{2} c^{2}} \sin ^{2} \theta, \\
& P_{\text {rad }}^{\mathrm{L}}\left(\varphi, \theta ; \omega_{s}\right)=J_{0} \frac{\omega_{s}\left[\left(\omega_{s} / c\right)^{2}-m^{2}\right]^{1 / 2}}{8 \varepsilon_{0} \pi^{2} c^{2}} \cos ^{2} \theta .
\end{aligned}
$$

The total radiated power in a Proca wave is the sum of the $\mathrm{T}$ and $\mathrm{L}$ contributions, i.e., $P_{\mathrm{rad}}\left(\varphi, \theta ; \omega_{s}\right)=P_{\mathrm{rad}}^{\mathrm{T}}\left(\varphi, \theta ; \omega_{s}\right)+$ $P_{\operatorname{rad}}^{\mathrm{L}}\left(\varphi, \theta ; \omega_{s}\right)$, which is a consequence of the fact that in a

\footnotetext{
${ }^{11}$ We also replaced $\hat{k}$ defined by (66) by the spherical angles $\varphi$ and $\theta$ in the arguments of $P^{\mathrm{T}}$ and $P^{\mathrm{L}}$ in order to make the Proca radiator power pattern similar to the traditional form familiar from antenna theory [84].
} 


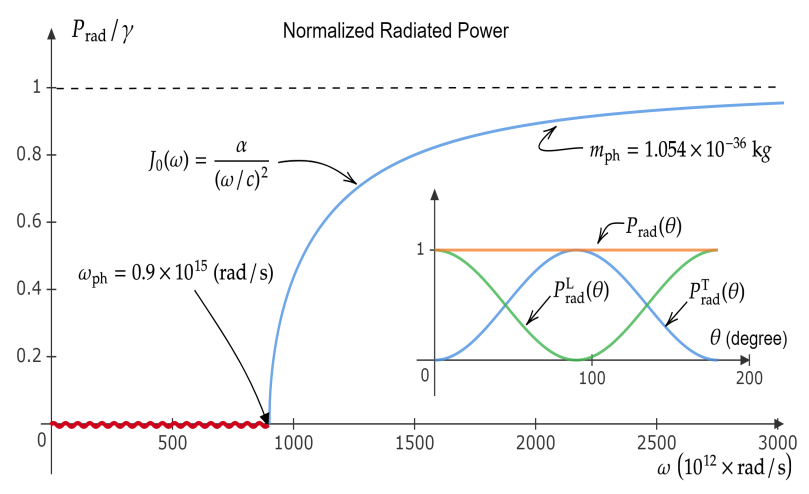

Fig. 11. Radiated power of an electrically-small dipole embedded into a Proca MTM. For simplicity, we denote $\omega_{s}$ by $\omega$. The radiated power normalization factor is $\gamma=\alpha\left(2 \varepsilon_{0} \pi c\right)^{-1}$, where the current source frequency profile is assumed to be $J_{0}(\omega)=\alpha(\omega / c)^{-2}$. The angular power radiation patterns of the T, L, and total fields are shown in the inset. The frequency interval $\left[0, \omega_{\mathrm{ph}}\right]$ highlighted by the wavy line is the antenna stopband while $\omega_{\mathrm{ph}}$ is the cutoff frequency.

Proca wave the $\mathrm{T}$ and $\mathrm{L}$ modes form a unity and are always excited simultaneously. ${ }^{12}$ Therefore, from (61) and (62) we find:

$$
P_{\mathrm{rad}}\left(\varphi, \theta ; \omega_{s}\right)=J_{0}\left(\omega_{s}\right) \frac{\omega_{s}\left[\left(\omega_{s} / c\right)^{2}-m^{2}\right]^{1 / 2}}{8 \varepsilon_{0} \pi^{2} c^{2}},
$$

where we have reemphasized that the current amplitude $J_{0}$ is a function of the source frequency $\omega_{s}$. The total radiated power through a full angular span is then:

$$
P_{\mathrm{rad}}=\int_{4 \pi} \mathrm{d} \Omega P_{\mathrm{rad}}(\Omega)=\frac{J_{0} \omega_{s} \sqrt{\left(\omega_{s} / c\right)^{2}-m^{2}}}{2 \varepsilon_{0} \pi c^{2}} .
$$

We have proved then the following fundamental theorems in Proca antenna theory:

Theorem V.1. The radiation power pattern of a single timeharmonic infinitesimal dipole source embedded into a Proca MTM is perfectly isotropic. Moreover, the radiated power density per solid angles is given by the expression (63).

This remarkable result represents a departure from classical (local) antenna theory where the source is embedded into either free space or a temporally dispersive - i.e., local domain. In the classical theory case, such perfect isotropic radiators are not possible due to the hairy ball theorem of differential and algebraic topology [103]. Nonlocal antenna theory generally predicts the possibility of perfect isotropic radiators if certain design conditions are met [55]. On the other hand, Theorem V.1 indicates that a Proca MTM represents a possible direct physical realization of such design conditions. In other words, designing a Proca MTM implies the design of a perfect isotropic radiator. It should be noted though that the above conclusion applies to individual Proca sources. If multiple Proca dipoles are placed in the vicinity of each other, then an array effect is observable [85] and the Proca antenna system can be made directive by controlling the relative excitations of each (isotropic) radiator.

\footnotetext{
${ }^{12}$ See Sec. IV and Theorem IV.1.
}

Fig. 11 shows an example of a Proca antenna radiation characteristics calculation plotted against frequency. We use the same example of an optical Proca MTM given in Fig. 1. For the practical estimation of the radiated power, a realistic current source frequency law is used with $J(\omega)=\alpha(\omega / c)^{-2}$, where $\alpha \in \mathbb{C}$ is a constant complex number and $\omega_{s}$ was rewritten as $\omega$ for simplicity. For sources with slower frequency decay such as constant current $J(\omega)=\alpha$ or $J(\omega)=$ $\alpha(\omega / c)^{-1}$, the radiated power diverges with frequency [55]. In practice, the current on any physically realizable antenna always strongly decays away from the central operational frequency band (resonance frequency for example in resonanttype antennas, see [85]). We show the power normalized to the peak current source level $\alpha$. The Proca point dipole antenna would not radiate unless the operating frequency exceeds the cutoff frequency $\omega_{\mathrm{ph}}=900 \times 10^{12} \mathrm{rad} / \mathrm{s}$ and within the lower frequency range we have an effective antenna radiation stopband. The angular power patterns are shown in the inset of Fig. 11 and they demonstrate the complementary relation between the $\mathrm{T}$ and $\mathrm{L}$ radiation fields. The combined pattern of the Proca source is perfectly isotropic (flat) with angles $\theta$.

\section{CONCLUSION}

We presented a complete theory of Proca metamaterials (Proca MTMs) and Proca waves starting from first principles. An exact equivalence of Proca-Maxwell theory and a special nonlocal metamaterial was reported and proved using a rigorous field theoretic approach. The obtained Proca MTM dielectric profile was then investigated in depth and the properties of Proca waves derived and discussed. Proca waves were found to constitute a distinct type of electromagnetic modes where both the longitudinal and transverse submodes have identical dispersion laws and propagate as a holistic unit while their quantization leads to massive photons. We presented some case studies of Proca MTMs analysis and design and also outlined practical applications to Proca antennas as an example of the emerging nonlocal technology antenna. It was found that single Proca dipole antennas are perfectly isotropic radiators (with respect to total power patterns), in contrast to classical antennas and other types of nonlocal antennas.

\section{APPENDIX A \\ ON THE LIMIT $m \rightarrow 0$}

The limit $m \rightarrow 0$ involves theoretical problems reminiscent of the limit $k \rightarrow 0$ in the theory of spatially dispersive domains [50]. Naturally, we expect that once $m=0$ in our expressions, all equations based on Maxwell-Proca equations should reduce to Maxwell's equations [7]. However, this is not always the case. For example, the dispersion relations in Proca MTMs do reduce to those of vacuum Maxwell's theory when $m \rightarrow 0$ but not the dielectric or conductivity tensor in general, e.g., the Proca MTM tensor. Indeed, if we take the limit $m \rightarrow 0$ in (28), we notice that the result is not the identity operator $\overline{\mathbf{I}}$. The nonlocal term proportional to $\hat{k} \hat{k}$ appears to persist even when the photon mass is manually set to zero. This is not surprising given that the origin of this term is really (22), which is inherently non-Maxwellian in vacuum and cannot be 
used in the case of $m=0$. This is also clear from the relation (25) where we get $\mathbf{E}=0$ if $m$ was manually set to zero there, obviously a nonphysical result since the electric field should not vanish in Maxwell's theory.

Consequently, the limit $m \rightarrow 0$ cannot be taken directly in our theory (at least for some expressions like those quoted above) and a further investigation is needed using more rigorous technique. This difficulty in Proca theories is well known and there exists several methods to deal with it and has been repeatedly addressed in quantum field theory [7]. One option is to study the structure of the Proca MTM tensor (28) in the complex plane with analytic continuation applied. This brings the open problem of whether the photon mass is really purely real. If $m$ is allowed to become complex, then the limit $m \rightarrow 0$ can be eliminated since $m$ may be replaced by $m+\mathrm{i} 0$, and the limit is then taken in the complex plane instead of the real axis $\operatorname{Re}\{m\}$. Full analysis along these lines is outside the scope of the present paper.

\section{APPENDIX B}

\section{Nonlocal Proca Antenna Theory}

In what follows we concentrate on the isotropic nonlocal MTM case because it is the only one relevant to Proca antenna theory. More general analysis can be found [54].

1) The General Setting: For a source with current distribution $\mathbf{J}_{\text {ant }}\left[\mathbf{k}, \omega_{l}(\mathbf{k}]\right.$ The momentum-space energy density function can be expressed by the general formula [54]:

$$
U_{l}(\mathbf{k})=\frac{1}{\varepsilon_{0}} R_{l}(\mathbf{k}) \mathbf{J}_{\text {ant }}^{*}\left[\mathbf{k}, \omega_{l}(\mathbf{k})\right] \cdot(\overline{\mathbf{I}}-\hat{k} \hat{k}) \cdot \mathbf{J}_{\text {ant }}\left[\mathbf{k}, \omega_{l}(\mathbf{k})\right],
$$

where $l$ is the modal index and $\omega_{l}=\omega_{l}(\mathbf{k})$ is the dispersion law of the $l$ th mode. Here, the spherical coordinates form of $\hat{k}$ is given by

$$
\hat{k}=\hat{k}(\Omega)=\hat{x} \cos \varphi \sin \theta+\hat{y} \cos \varphi \sin \theta+\hat{z} \cos \theta,
$$

where $\Omega:=(\theta, \varphi)$. The most important quantity for computing the radiation energy/power profile are the $R(k)$ functions [54]

$$
\begin{gathered}
R_{l}^{\mathrm{L}}(k)=\left.\frac{1}{\omega \frac{\partial \varepsilon^{\mathrm{L}}(k, \omega)}{\partial \omega}}\right|_{\omega=\omega_{\mathrm{L}, l}(k)}, \\
R_{l}^{\mathrm{T}}(\mathbf{k})=\left.\frac{1}{\omega \frac{\partial}{\partial \omega}\left[\varepsilon^{\mathrm{T}}(k, \omega)-n^{2}(k, \omega)\right]}\right|_{\omega=\omega_{\mathrm{T}, l}(k)},
\end{gathered}
$$

where $n=c^{2} k^{2} / \omega^{2}$ is the index of refraction while $\omega_{\mathrm{L} / \mathrm{T}, l}$ are the $\mathrm{L} / \mathrm{T}$ dispersion relations of the $\mathrm{L} / \mathrm{T} l$ th modes, respectively.

2) Transverse (T) Antenna Radiation Pattern Formulas: After summing over the degeneracy degrees of freedom in transverse polarized modes, it can be shown that the radiation energy density acquires the compact form [54]

$$
U_{l}^{\mathrm{T}}(\mathbf{k})=\frac{1}{\varepsilon_{0}} R_{l}(\mathbf{k})\left|\hat{k} \times \mathbf{J}_{\text {ant }}\left[\mathbf{k}, \omega_{l}(\mathbf{k})\right]\right|^{2},
$$

Next, a key idea proposed in [74] was to use dispersion relations to express the $U(\mathbf{k})$ function above in terms angles (in space) and frequency, i.e., in the form $U_{l}^{\mathrm{T}}(\omega, \Omega)$ which is defined by the equality [55], [74]:

$$
\int \frac{\mathrm{d}^{3} k}{(2 \pi)^{3}} U_{l}(\mathbf{k})=\int \mathrm{d} \omega \int \mathrm{d} \Omega U_{l}(\omega, \Omega) .
$$

It can be shown after some manipulations that the final expression is [55]:

$$
U_{l}^{\mathrm{T}}(\omega, \hat{k})=\frac{k_{\mathrm{T}, l}^{2}(\omega)}{(2 \pi)^{3}} \frac{\mathrm{d} k_{\mathrm{T}, l}(\omega)}{\mathrm{d} \omega} U_{\mathrm{T}, l}\left[k_{\mathrm{T}, l}(\omega), \hat{k}\right] .
$$

Physically, the quantity (71) represents the radiation energy density, or energy per unit solid angle per unit radian frequency (Watt per starad per $\mathrm{rad} / \mathrm{s}$ ). Using (69) in (71), the final $\mathrm{T}$ wave formula is

$$
U^{\mathrm{T}}(\omega, \hat{k})=\frac{k_{\mathrm{T}}^{2}(\omega) R_{\mathrm{T}}(\omega)}{\varepsilon_{0}(2 \pi)^{3}} \frac{\mathrm{d} k_{\mathrm{T}}(\omega)}{\mathrm{d} \omega}\left|\hat{k} \times \mathbf{J}_{\text {ant }}\left[k_{\mathrm{T}}(\omega), \hat{k}\right]\right|^{2},
$$

with detailed derivation outlined in [54] and [55].

3) Longitudinal (L) Antenna Radiation Pattern Formulas: The procedure for $\mathrm{L}$ wave radiation is parallel to that of the $\mathrm{T}$ wave case. The general formula for the $U$-function in momentum space is:

$$
U_{l}(\mathbf{k})=\frac{1}{\varepsilon_{0}} R_{l}(\mathbf{k})\left|\hat{k} \cdot \mathbf{J}_{\text {ant }}\left[\mathbf{k}, \omega_{l}(\mathbf{k})\right]\right|^{2} .
$$

The corresponding frequency-dependent radiation energy is given by:

$$
U_{\mathrm{L}}(\omega, \hat{k})=\frac{k_{\mathrm{L}}^{2}(\omega)}{\varepsilon_{0}(2 \pi)^{3}} \frac{\mathrm{d} k_{\mathrm{L}}(\omega)}{\mathrm{d} \omega} R^{\mathrm{L}}(\omega)\left|\hat{k} \cdot \mathbf{J}_{\mathrm{ant}}\left[k_{\mathrm{L}}(\omega), \hat{k}\right]\right|^{2} .
$$

The derivation of (74) utilizes the same definition (70) and the transformation of variables enacted via the dispersion relation utilized in the $\mathrm{T}$ wave case of Appendix B-2.

4) Computing the $T$ and L Proca Antenna Radiation Pattern: We now calculate the $\mathrm{T}$ and $\mathrm{L}$ radiation formulas (72) and (74) for the specific case of the nonlocal Proca medium with $\mathrm{T}$ and $\mathrm{L}$ dielectric function profiles (30) and (31), respectively. First, note that a characteristic feature of Proca waves (Theorem IV.1) is that both of the L and T waves share the same dispersion relation, namely (41), which considerably simplifies the calculations of Proca antenna theory. In particular, there is no need to differentiate between $\mathrm{L}$ and $\mathrm{T}$ modes and we drop the super/sub-scripts in expressions like $\omega_{\mathrm{L}}$ and $\omega_{\mathrm{T}}$. However, the distinction between $R^{\mathrm{L}}(\omega)$ and $R^{\mathrm{T}}(\omega)$ remains valid since the $\mathrm{L}$ and $\mathrm{T}$ dielectric functions in Proca MTMs are distinct for any nonzero $k$. Remarkably though, it turns out they are both equal to each other as we now prove the following theorem:

Theorem B.1. In Proca MTMs, the momentum-space radiation $L$ and $T$ wave functions $R^{\mathrm{L}}(\omega)$ and $R^{\mathrm{T}}(\omega)$ satisfy

$$
R^{\mathrm{L}}(\omega)=R^{\mathrm{L}}(\omega)=\frac{1}{2} .
$$

Proof. We prove the L wave case first. The formula (67) will be used so we need to first compute

$$
\omega \frac{\partial}{\partial \omega} \varepsilon^{L}(k, \omega)=\omega \frac{\partial}{\partial \omega}\left[1-\frac{\omega_{\mathrm{p}}^{2}(k)}{\omega^{2}}\right]=\frac{2 \omega_{\mathrm{p}}^{2}(k)}{\omega^{2}},
$$


where the L profile expression (31) was utilized. Substituting into (67), we deduce

$$
R^{\mathrm{L}}(k)=\frac{\omega^{2}(k)}{2 \omega_{\mathrm{p}}^{2}(k)}=\frac{1}{2},
$$

where $k=k(\omega)$ (dispersion law) and the second equality follows from (32) and (41). Therefore, $R^{\mathrm{L}}(\omega)=1 / 2$ as well by noting that $R^{\mathrm{T}}(\omega)=R^{\mathrm{T}}[k(\omega)]$.

For the $\mathrm{T}$ wave case, we employ the formula (68), so we need to evaluate the quantity $\omega \partial / \partial \omega\left[\varepsilon^{\mathrm{T}}(k, \omega)-n^{2}\right]$ as follows:

$$
\omega \frac{\partial}{\partial \omega}\left[1-\frac{\omega_{\mathrm{p}}^{2}(0)}{\omega^{2}}-\frac{c^{2} k^{2}}{\omega^{2}}\right]=\frac{2\left[\omega_{\mathrm{p}}^{2}(0)+c^{2} k^{2}\right]}{\omega^{2}},
$$

where the T dielectric profile (30) was used. Substituting into (68), we find

$$
R^{\mathrm{T}}(k)=\frac{\omega^{2}(k)}{2\left[c^{2} m^{2}+c^{2} k^{2}\right]}=\frac{1}{2},
$$

where $k=k(\omega)$ (dispersion law) and the second equality follows from (32). Therefore, $R^{\mathrm{T}}(\omega)=1 / 2$ as well by noting that $R^{\mathrm{T}}(\omega)=R^{\mathrm{T}}[k(\omega)]$.

From this theorem, the relations (72) and (74) reduces into

$$
\begin{aligned}
U^{\mathrm{T}}(\omega, \hat{k}) & =\frac{\omega\left[(\omega / c)^{2}-m^{2}\right]^{1 / 2}}{2 \varepsilon_{0}(2 \pi)^{3} c^{2}}|\hat{k} \times \mathbf{J}[k(\omega), \hat{k}]|^{2}, \\
U^{\mathrm{L}}(\omega, \hat{k}) & =\frac{\omega\left[(\omega / c)^{2}-m^{2}\right]^{1 / 2}}{2 \varepsilon_{0}(2 \pi)^{3} c^{2}}|\hat{k} \cdot \mathbf{J}[k(\omega), \hat{k}]|^{2},
\end{aligned}
$$

which give the $\mathrm{T}$ and $\mathrm{L}$ energy density patterns of an arbitrary current source embedded into a Proca MTM.

\section{REFERENCES}

[1] I. Y. Kobzarev and L. B. Okun, "On the photon mass," Soviet Physics Uspekhi, vol. 11, no. 3, pp. 338-341, March 1968.

[2] P. Robles and F. Claro, "Can there be massive photons? a pedagogical glance at the origin of mass," European Journal of Physics, vol. 33, no. 5, pp. 1217-1226, jul 2012.

[3] M. Gondran, "The Proca equations derived from first principles," American Journal of Physics, vol. 77, no. 10, pp. 925-926, Oct. 2009.

[4] C. Marchal, "LIGO: The hypothesis of photons with non-zero rest mass," Journal of Physics: Conference Series, vol. 1251, p. 012034, jun 2019.

[5] J. Jackson, Classical electrodynamics. New York: Wiley, 1999.

[6] C. Brau, Modern problems in classical electrodynamics. New York: Oxford University Press, 2004.

[7] S. Coleman, Quantum field theory: lectures of Sidney Coleman. New Jersey: World Scientific, 2019.

[8] W. Greiner and J. Reinhardt, Field quantization. Berlin New York: Springer, 1996.

[9] A. M. Gabovich and N. A. Gabovich, "How to explain the non-zero mass of electromagnetic radiation consisting of zero-mass photons," European Journal of Physics, vol. 28, no. 4, pp. 649-655, May 2007.

[10] L.-C. Tu, J. Luo, and G. T. Gillies, "The mass of the photon," Reports on Progress in Physics, vol. 68, no. 1, pp. 77-130, nov 2005.

[11] A. S. Goldhaber and M. M. Nieto, "Photon and graviton mass limits," Rev. Mod. Phys., vol. 82, pp. 939-979, Mar 2010.

[12] V. Errasti Díez, B. Gording, J. A. Méndez-Zavaleta, and A. SchmidtMay, "Complete theory of Maxwell and Proca fields," Phys. Rev. D, vol. 101, p. 045008, Feb 2020.

[13] _ - "Maxwell-Proca theory: Definition and construction," Phys. Rev. $D$, vol. 101, p. 045009, Feb 2020.

[14] A. J. Silenko, "Relativistic quantum mechanics of a Proca particle in riemannian spacetimes," Phys. Rev. D, vol. 98, p. 025014, Jul 2018.
[15] A. González, R. Linares, M. Maceda, and O. Sánchez-Santos, "Noncommutative Einstein-Proca spacetime," Phys. Rev. D, vol. 90, p. 124085, Dec 2014.

[16] N. Sanchis-Gual, C. Herdeiro, E. Radu, J. C. Degollado, and J. A. Font, "Numerical evolutions of spherical Proca stars," Phys. Rev. D, vol. 95, p. 104028, May 2017.

[17] S. Nakamura, R. Kase, and S. Tsujikawa, "Cosmology in beyondgeneralized Proca theories," Phys. Rev. D, vol. 95, p. 104001, May 2017.

[18] B. Bertrand and P. Defraigne, "A model of massive electromagnetism for the detection of topological dark matter through GNSS networks," in 2018 Conference on Precision Electromagnetic Measurements (CPEM 2018), 2018, pp. 1-2.

[19] D. D. Ryutov, "Using plasma physics to weigh the photon," Plasma Physics and Controlled Fusion, vol. 49, no. 12B, pp. B429-B438, nov 2007.

[20] A. Accioly, J. Helayël-Neto, R. Turcati, J. Morais, and E. Scatena, "Gravitational and quantum bounds on the photon mass," Classical and Quantum Gravity, vol. 27, no. 20, p. 205010, sep 2010.

[21] L. A. Rivlin, "Nonzero-mass photons: from the wave equation to the schrödinger equation," Quantum Electronics, vol. 23, no. 9, pp. 799 804, sep 1993

[22] — "Is the photon mass zero?" Soviet Journal of Quantum Electronics, vol. 22, no. 8, pp. 771-773, aug 1992.

[23] R. Mattuck, A guide to Feynman diagrams in the many-body problem. New York: Dover Publications, 1992.

[24] D. Pines, The many-body problem. Reading, Mass: Addison-Wesley, 1997.

[25] V. S. Gorelik, "Effective mass of photons and the existence of heavy photons in photonic crystals," Physica Scripta, vol. T140, p. 014046 , Sep. 2010.

[26] W. Tan, Y. Sun, H. Chen, and S.-Q. Shen, "Photonic simulation of topological excitations in metamaterials," Scientific Reports, vol. 4, no. 1, Jan 2014.

[27] A. Alberucci, C. P. Jisha, A. D. Boardman, and G. Assanto, "Anomalous diffraction in hyperbolic materials," Phys. Rev. A, vol. 94, p. 033830, Sep 2016.

[28] I. Smolyaninov, Metamaterial multiverse. Morgan \& Claypool Publishers,IOP Publishing, 2018.

[29] O. M. P. Bilaniuk, V. K. Deshpande, and E. C. G. Sudarshan, "Meta relativity," American Journal of Physics, vol. 30, no. 10, pp. 718-723, Oct. 1962.

[30] O. M. Bilaniuk and E. C. G. Sudarshan, "Causality and space-like signals," Nature, vol. 223, no. 5204, pp. 386-387, Jul. 1969.

[31] C. Z. Tan, "Imaginary rest mass of a photon in a dispersive medium," Optik, vol. 126, no. 24, pp. 5304 - 5306, 2015.

[32] D. Park, "Plasma dynamics and the photon mass," Journal of Physics A: Mathematical, Nuclear and General, vol. 6, no. 12, pp. 1949-1953, dec 1973.

[33] J. T. Mendonça, A. M. Martins, and A. Guerreiro, "Field quantization in a plasma: Photon mass and charge," Phys. Rev. E, vol. 62, pp. 29892991, Aug 2000.

[34] F. A. Asenjo, V. Muñoz, and J. A. Valdivia, "Relativistic mass and charge of photons in thermal plasmas through electromagnetic field quantization," Phys. Rev. E, vol. 81, p. 056405, May 2010.

[35] F. Tamburini, A. Sponselli, B. Thidé, and J. T. Mendonça, "Photon orbital angular momentum and mass in a plasma vortex," EPL (Europhysics Letters), vol. 90, no. 4, p. 45001, may 2010.

[36] Z. Cheng, "Optical analogue of the polaron: Dressed photon in a nonlinear fabry-pérot cavity," EPL (Europhysics Letters), vol. 101, no. 1, p. 14003, Jan 2013.

[37] J.-Q. Shen, "Frequency-independent effective rest mass of photons in the two time derivative lorentz material model," Journal of Optics A: Pure and Applied Optics, vol. 6, no. 2, pp. 239-245, Jan 2004.

[38] B. T. L. Quyen, D. N. Mai, N. T. Lan, and N. A. Viet, "Effective mass of photon in liquid water environment of life and soft maters," Journal of Physics: Conference Series, vol. 627, p. 012015, jun 2015.

[39] V. S. Gorelik, "Massive photon properties in 3D photonic crystals, filled by dielectrics or metals," Physica Scripta, vol. T135, p. 014039, Jul 2009.

[40] J.-M. André and P. Jonnard, "Effective mass of photons in a onedimensional photonic crystal," Physica Scripta, vol. 84, no. 3, p. 035708, sep 2011.

[41] P. Mati, "Statistical theory of photon gas in plasma," Journal of Statistical Mechanics: Theory and Experiment, vol. 2020, no. 2, p. 023102, Feb 2020. 
[42] W. Heisenberg and H. Euler, "Folgerungen aus der diracschen theorie des positrons," Z. Physik, vol. 98, no. 11-12, pp. 714-732, Nov. 1936.

[43] J. M. Jauch and K. M. Watson, "Phenomenological quantumelectrodynamics," Phys. Rev., vol. 74, pp. 950-957, Oct 1948.

[44] G. V. Dunne, "The Heisenberg-Euler effective action: 75 years on," International Journal of Modern Physics A, vol. 27, no. 15, p. 1260004, 2012.

[45] R. Battesti and C. Rizzo, "Magnetic and electric properties of a quantum vacuum," Reports on Progress in Physics, vol. 76, no. 1, p. 016401, dec 2012.

[46] L. D. Landau, The classical theory of fields. Oxford Boston: Butterworth Heinemann, 2000.

[47] S. Pekar, "The theory of electromagnetic waves in a crystal in which excitons are produced," JETP, vol. 6, no. 4, p. 785, May 1957.

[48] V. Ginzburg, "Electromagnetic waves in isotropic and crystalline media characterized by dielectric permittivity with spatial dispersion," JETP, vol. 7, no. 6, p. 1096, December 1958.

[49] S. Mikki and A. Kishk, "Nonlocal electromagnetic media: A paradigm for material engineering," in Passive Microwave Components and Antennas. InTech, April 2010.

[50] V. Agranovich and V. Ginzburg, Crystal optics with spatial dispersion, and excitons. Berlin, Heidelberg: Springer Berlin HeidelbergImprint Springer, 1984.

[51] S. Mikki and Y. Antar, New Foundations for Applied Electromagnetics: The Spatial Structure of Fields. London: Artech House, 2016.

[52] V. L. Ginzburg, The propagation of electromagnetic waves in plasmas. Oxford,New York: Pergamon Press, 1970.

[53] S. Mikki and A. Kishk, "Electromagnetic wave propagation in nonlocal media: Negative group velocity and beyond," Progress In Electromagnetics Research B, vol. 14, pp. 149-174, 2009.

[54] S. Mikki, "Theory of electromagnetic radiation in nonlocal metamaterials - Part I: Foundations," Progress In Electromagnetics Research B, vol. 89 , pp. 63-86, 2020.

[55] — - "Theory of electromagnetic radiation in nonlocal metamaterials - Part II: Applications," Progress In Electromagnetics Research B, vol. 89, pp. 87-109, 2020.

[56] S. Mikki and A. Kishk, "Theory of optical scattering by carbon nanotubes," Microwave and Optical Technology Letters, vol. 49, no. 10, pp. 2360-2364, Jul. 2007.

[57] S. M. Mikki and A. A. Kishk, "Electromagnetic scattering by multiwall carbon nanotubes using effective-boundary condition approach," in 2008 IEEE Antennas and Propagation Society International Symposium, July 2008, pp. 1-4.

[58] — - "Exact derivation of the dyadic green's functions of carbon nanotubes using microscopic theory," in 2007 IEEE Antennas and Propagation Society International Symposium, June 2007, pp. 4332 4335

[59] S. Mikki and A. Kishk, "Electromagnetic scattering by multi-wall carbon nanotubes," Progress In Electromagnetics Research B, vol. 17, pp. 49-67, 2009.

[60] — "A symmetry-based formalism for the electrodynamics of nanotubes," Progress In Electromagnetics Research, vol. 86, pp. 111-134, 2008.

[61] _ - "Derivation of the carbon nanotube susceptibility tensor using lattice dynamics formalism," Progress In Electromagnetics Research B, vol. 9, pp. 1-26, 2008.

[62] — - "An efficient algorithm for the analysis and design of carbon nanotube photonic crystals," Progress In Electromagnetics Research $C$, vol. 83, pp. 83-96, 2018.

[63] S. Mikki and A. Kishk, "Effective medium theory for carbon nanotube composites and their potential applications as metamaterials," in 2007 IEEE/MTT-S International Microwave Symposium, June 2007, pp. $1137-1140$.

[64] — "Mean-field electrodynamic theory of aligned carbon nanotube composites," IEEE Transactions on Antennas and Propagation, vol. 57, no. 5, pp. 1412-1419, May 2009.

[65] S. Mikki and Y. Antar, "On electromagnetic radiation in nonlocal environments: Steps toward a theory of near field engineering," in 2015 9th European Conference on Antennas and Propagation (EuCAP), April 2015, pp. 1-5.

[66] S. I. Pekar, Crystal optics and additional light waves. Menlo Park, Calif: Benjamin/Cummings Pub. Co, 1983

[67] Y. A. Ilinskii and L. Keldysh, Electromagnetic response of material media. New York: Springer Science+Business Media, 1994.

[68] V. L. Ginzburg, Theoretical physics and astrophysics. Oxford New York: Pergamon Press, 1979.
[69] L. Felsen, Radiation and scattering of waves. Piscataway, NJ: IEEE Press, 1994.

[70] W. C. Chew, Waves and fields in inhomogenous media. Wiley-IEEE, 1999.

[71] L. D. Landau and E. Lifshitz, Electrodynamics of continuous media. Oxford England: Butterworth-Heinemann, 1984.

[72] R. Penrose, The road to reality: a complete guide to the laws of the universe. New York: Vintage Books, 2007.

[73] _- Cycles of time: an extraordinary new view of the universe. New York: Vintage Books, 2012.

[74] S. Mikki, "Exact derivation of the radiation law of antennas embedded into generic nonlocal metamaterials: A momentum-space approach," in 2020 14th European Conference on Antennas and Propagation (EuCAP), 2020, pp. 1-5.

[75] D. Bohm, The special theory of relativity. London New York: Routledge, 2006

[76] O. A. Peverini, R. Tascone, G. Addamo, G. Virone, and R. Orta, "On superluminal propagation," IEEE Antennas and Wireless Propagation Letters, vol. 7, pp. 101-104, 2008.

[77] D. R. Solli, C. F. McCormick, R. Y. Chiao, and J. M. Hickmann, "Experimental observation of superluminal group velocities in bulk two-dimensional photonic bandgap crystals," IEEE Journal of Selected Topics in Quantum Electronics, vol. 9, no. 1, pp. 40-42, 2003.

[78] D. F. Sievenpiper, "Superluminal waveguides based on non-Foster circuits for broadband leaky-wave antennas," IEEE Antennas and Wireless Propagation Letters, vol. 10, pp. 231-234, 2011.

[79] D. Oloumi and K. Rambabu, "Studying the superluminal behavior of UWB antennas and its effect on near-field imaging," IEEE Transactions on Antennas and Propagation, vol. 64, no. 12, pp. 5084-5093, 2016.

[80] R. S. C. Winter, D. Oloumi, and K. Rambabu, "UWB sensor characterization for radar sensing and imaging in superluminal propagation regions," IEEE Transactions on Microwave Theory and Techniques, pp. $1-1,2020$.

[81] P. Milonni, Fast light, slow light, and left-handed light. Bristol Philadelphia: Institute of Physics, 2005.

[82] R. N. C. Pfeifer, T. A. Nieminen, N. R. Heckenberg, and H. Rubinsztein-Dunlop, "Colloquium: Momentum of an electromagnetic wave in dielectric media," Rev. Mod. Phys., vol. 79, pp. 1197 1216, Oct 2007.

[83] I. Brevik, "Analysis of recent interpretations of the AbrahamMinkowski problem," Phys. Rev. A, vol. 98, p. 043847, Oct 2018

[84] S. A. Schelkunoff and H. T. Friss, Antennas: Theory and practice. New York; Chapman \& Hall: London, 1952.

[85] C. A. Balanis, Antenna Theory: Analysis and Design, 4th ed. Interscience: Wiley, 2015.

[86] S. Mikki and A. Kishk, "Theory and Applications of Infinitesimal Dipole Models for Computational Electromagnetics," IEEE Transactions on Antennas and Propagation, vol. 55, no. 5, pp. 1325-1337, May 2007

[87] S. Mikki and Y. Antar, "Near-field analysis of electromagnetic interactions in antenna arrays through equivalent dipole models," IEEE Transactions on Antennas and Propagation, vol. 60, no. 3, pp. 13811389, March 2012.

[88] S. Clauzier, S. Mikki, and Y. Antar, "A generalized methodology for obtaining antenna array surface current distributions with optimum cross-correlation performance for mimo and spatial diversity applications," IEEE Antennas and Wireless Propagation Letters, vol. 14, pp. 1451-1454, March 2015.

[89] S. M. Mikki and Y. M. M. Antar, "A new technique for the analysis of energy coupling and exchange in general antenna systems," IEEE Transactions on Antennas and Propagation, vol. 63, no. 12, pp. 55365547, Dec 2015

[90] S. Mikki and Y. Antar, "On the Fundamental Relationship Between the Transmitting and Receiving Modes of General Antenna Systems: A New Approach," IEEE Antennas and Wireless Propagation Letters, vol. 11, pp. 232-235, 2012.

[91] D. Sarkar, S. Mikki, K. V. Srivastava, and Y. Antar, "Dynamics of antenna reactive energy using time-domain IDM method," IEEE Transactions on Antennas and Propagation, vol. 67, no. 2, pp. 10841093, Feb 2019.

[92] S. Mikki and Y. Antar, "A rigorous approach to mutual coupling in general antenna systems through perturbation theory," IEEE Antennas and Wireless Communication Letters, vol. 14, pp. 115-118, 2015.

[93] S. Mikki, S. Clauzier, and Y. Antar, "Empirical geometrical bounds on MIMO antenna arrays for optimum diversity gain performance: An electromagnetic design approach," IEEE Access, vol. 6, pp. 39876 39894, 2018. 
[94] S. Mikki, D. Sarkar, and Y. Antar, "Near-field cross-correlation analysis for MIMO wireless communications," IEEE Antennas and Wireless Propagation Letters, vol. 18, no. 7, pp. 1357-1361, July 2019.

[95] S. Mikki, S. Clauzier, and Y. Antar, "A correlation theory of antenna directivity with applications to superdirective arrays," IEEE Antennas and Wireless Propagation Letters, vol. 18, no. 5, pp. 811-815, May 2019.

[96] S. Clauzier, S. Mikki, A. Shamim, and Y. Antar, "A new method for the design of slot antenna arrays: Theory and experiment," in 2016 10th European Conference on Antennas and Propagation (EuCAP), April 2016, pp. 1-5.

[97] S. Clauzier, S. Mikki, and Y. Antar, "Design of high-diversity gain MIMO antenna arrays through surface current optimization," in 2015 IEEE International Symposium on Antennas and Propagation USNC/URSI National Radio Science Meeting, July 2015, pp. 9-10.

[98] _ _ "Design of near-field synthesis arrays through global optimization," IEEE Transactions on Antennas and Propagation, vol. 63, no. 1, pp. 151-165, Jan 2015.

[99] S. Mikki, S. Clauzier, M. Karimi, A. Shamim, and Y. Antar, "Slot antenna array synthesis using the infinitesimal dipole model technique: Theory and experiment (forthcoming)," International Journal of RF and Microwave Computer-Aided Engineering, 2019.

[100] S. Mikki and Y. Antar, "On cross correlation in antenna arrays with applications to spatial diversity and MIMO systems," IEEE Transactions on Antennas and Propagation, vol. 63, no. 4, pp. 1798-1810, April 2015.

[101] A. Alzahed, S. Mikki, and Y. Antar, "Nonlinear mutual coupling compensation operator design using a novel electromagnetic machine learning paradigm," IEEE Antennas and Wireless Propagation Letters, vol. 18 , no. 5 , pp. 861-865, 2019.

[102] R. Godement, Analysis II: differential and integral calculus, fourier series, holomorphic functions. Berlin: Springer-Verlag, 2005.

[103] K. Burns and M. Gidea, Differential geometry and topology: with a view to dynamical systems. Boca Raton: CRC Press, 2005. 